\title{
The Ensemble Kalman Filter: Theoretical Formulation and Practical Implementation
}

\author{
Geir Evensen \\ Nansen Environmental and Remote Sensing Center, Bergen Norway \\ The date of receipt and acceptance will be inserted by the editor
}

\begin{abstract}
The purpose of this paper is to provide a comprehensive presentation and interpretation of the Ensemble Kalman Filter (EnKF) and its numerical implementation. The EnKF has a large user group and numerous publications have discussed applications and theoretical aspects of it. This paper reviews the important results from these studies and also presents new ideas and alternative interpretations which further explain the success of the EnKF. In addition to providing the theoretical framework needed for using the EnKF, there is also a focus on the algorithmic formulation and optimal numerical implementation. A program listing is given for some of the key subroutines. The paper also touches upon specific issues such as the use of nonlinear measurements, in situ profiles of temperature and salinity, and data which are available with high frequency in time. An ensemble based optimal interpolation (EnOI) scheme is presented as a cost effective approach which may serve as an alternative to the EnKF in some applications.
\end{abstract}

Key words Data assimilation - Ensemble Kalman Filter

\section{Introduction}

The Ensemble Kalman Filter has been examined and applied in a number of studies since it was first introduced by Evensen (1994b). It has gained popularity because of it's simple conceptual formulation and relative ease of implementation, e.g., it requires no derivation of a tangent linear operator or adjoint equations and no integrations backward in time. Further, the computational requirements are affordable and comparable with other popular sophisticated assimilation methods such as the representer method by Bennett (1992); Bennett et al. (1993); Bennett and Chua (1994); Bennett et al.

Send offprint requests to: Geir Evensen, Nansen Environmental and Remote Sensing Center, Edvard Griegsvei 3A, 5059 Solheimsviken, Norway, e-mail: Geir.Evensen@nersc.no
(1996) and the 4DVAR method which has been much studied by the meteorological community (see e.g. Talagrand and Courtier, 1987; Courtier and Talagrand, 1987; Courtier et al., 1994; Courtier, 1997).

This paper gives a comprehensive presentation of the EnKF, and it may serve as an EnKF reference document. For a user of the EnKF it provides citations to hopefully all previous publications where the EnKF has been examined or used. It also provides a detailed presentation of the method both in terms of theoretical aspects and the practical implementation. For experienced EnKF users it will provide a better understanding of the EnKF through the presentation of a new and alternative interpretation and implementation of the analysis scheme.

In the next section, an overview is given of previous works involving the EnKF. Further, in Section 3, an overview of the theoretical formulation of the EnKF will be given. Thereafter the focus will be on implementation issues starting with the generation of the initial ensemble in Section 4.1 and the stochastic integration of the ensemble members in Section 4.2. The major discussion in this paper relates to the EnKF analysis scheme which is given in Section 4.3. Section 5 discusses particular aspects of the numerical implementation. Appendix A presents an approach for examining the consistency of the EnKF based on comparisons of innovations and predicted error statistics. In Appendix B an optimal interpolation algorithm is presented. It uses a stationary ensemble but is otherwise similar to the EnKF, and it can thus be denoted Ensemble Optimal Interpolation (EnOI). In Appendix $\mathrm{C}$ we have given an algorithm which is currently used for assimilation of observations of subsurface quantities. In Appendix D the Ensemble Kalman Smoother (EnKS) is presented in terms of the terminology developed in this paper. It is illustrated how the smoother solution can be very efficiently computed as a reanalysis following the use of the EnKF. Finally in Appendix E we have reviewed and detailed the presentation of the algorithm used for the generation of pseudo random fields. 


\section{Chronology of ensemble assimilation developments}

This section attempts to provide a complete overview of the developments and applications related to the EnKF. In addition it also points to other recently proposed ensemble based methods and some smoother applications.

\subsection{Applications of the EnKF}

Applications involving the EnKF are numerous and includes the initial work by Evensen (1994b) and an additional example in Evensen (1994a) which showed that the EnKF resolved the closure problems reported from applications of the Extended Kalman Filter (EKF).

An application with assimilation of altimeter data for the Agulhas region was discussed in Evensen and van Leeuwen (1996) and later in an intercomparison with the Ensemble Smoother (ES) by van Leeuwen and Evensen (1996).

An example with the Lorenz attractor was given by Evensen (1997) where it was shown that the EnKF could track the phase transitions and find a consistent solution with realistic error estimates even for such a chaotic and nonlinear model.

Burgers et al. (1998) reviewed and clarified some points related to the perturbation of measurements in the analysis scheme, and also gave a nice interpretation supporting the use of the ensemble mean as the best estimate.

Houtekamer and Mitchell (1998) introduced a variant of the EnKF where two ensembles of model states are integrated forward in time, and statistics from one ensemble is used to update the other. The use of two ensembles was motivated by claiming that this would reduce possible inbreeding in the analysis. This has, however, lead to some dispute discussed in the comment by van Leeuwen (1999a) and the reply by Houtekamer and Mitchell (1999).

Miller et al. (1999) included the EnKF in a comparison with nonlinear filters and the Extended Kalman Filter, and concluded that it performed well, but could be beaten by a nonlinear and more expensive filter in difficult cases where the ensemble mean is not a good estimator.

Madsen and Cañizares (1999) compared the EnKF and the reduced rank square root implementation of the Extended Kalman filter with a 2-D storm surge model. This is a weakly nonlinear problem and good agreement was found between the EnKF and the extended Kalman filter implementation.

Echevin et al. (2000) studied the EnKF with a coastal version of the Princeton Ocean Model and focussed in particular on the horizontal and vertical structure of multivariate covariance functions from sea surface height. It was concluded that the EnKF could capture anisotropic covariance functions resulting from the impact of coastlines and coastal dynamics, and had a particular advantage over simpler methodologies in such areas.

Evensen and van Leeuwen (2000) rederived the EnKF as a suboptimal solver for the general Bayesian problem of finding the posterior distribution given densities for the model prediction and the observations. From this formulation the general filter could be derived and the EnKF could be shown to be a suboptimal solver of the general filter where the prior densities are assumed to be Gaussian distributed.

Hamill and Snyder (2000) constructed a hybrid assimilation scheme by combining 3DVAR and the EnKF. The estimate is computed using the 3DVAR algorithm but the background covariance is a weighted average of the time evolving EnKF error covariance and the constant 3DVAR error covariance. A conclusion was that with increasing ensemble size the best results were found with larger weight on the EnKF error covariance.

Hamill et al. (2000) report from working groups in a workshop on ensemble methods.

Keppenne (2000) implemented the EnKF with a two layer shallow water model and examined the method in twin experiments assimilating synthetic altimetry data. He focused on the numerical implementation on parallel computers with distributed memory and found the approach efficient for such systems. He also examined the impact of ensemble size and concluded that realistic solutions could be found using a modest ensemble size.

Mitchell and Houtekamer (2000) introduced an adaptive formulation of the EnKF where the model error parameterization was updated by incorporating information from the innovations during the integration.

Park and Kaneko (2000) presented an experiment where the EnKF was used to assimilate acoustic tomography data into a barotropic ocean model.

Gronnevik and Evensen (2001) examined the EnKF for use in fish stock assessment, and also intercompared it with the Ensemble Smoother (ES) and the Ensemble Kalman Smoother (EnKS).

Heemink et al. (2001) have been examining different approaches which combine ideas from RRSQRT filtering and the EnKF to derive computationally more efficient methods.

Houtekamer and Mitchell (2001) have continued the examination of the two-ensemble approach and introduced a technique for computing the global EnKF analysis in the case with many observations, and also a method for filtering of eventual long range spurious correlations caused by a limited ensemble size. As will be seen below the current paper presents a much more efficient way to compute the global analysis and also argues against filtering of covariances.

Pham (2001) reexamined the EnKF in an application with the Lorenz attractor and intercompared results with those obtained from versions of the Singular Evolutive Extended Kalman (SEEK) filter and a particle filter. Ensembles with very few members were used and this favoured methods like the SEEK where the "ensemble" of EOFs is selected to best possible represent the model attractor.

Verlaan and Heemink (2001) applied the RRSQRT and EnKF filters in test examples with the purpose of classifying and defining a measure of the degree of nonlinearity of the model dynamics. Such an estimate may have an impact on the choice of assimilation method.

Hansen and Smith (2001) proposed a method for producing analysis ensembles based on integrated use of the 4DVAR 
method and the EnKF. A probabilistic approach was used and lead to high numerical cost, but an improved estimate could be found compared to 4DVAR and the EnKF used separately.

Hamill et al. (2001) examined the impact of ensemble size on noise in distant covariances. They evaluated the impact of using an "inflation factor" as introduced by Anderson and Anderson (1999), and also the use of a Schur product of the covariance with a correlation function to localize the background covariances as previously discussed by Houtekamer and Mitchell (2001). The inflation factor is used to replace the forecast ensemble according to

$$
\boldsymbol{\psi}_{j}=\rho\left(\boldsymbol{\psi}_{j}-\overline{\boldsymbol{\psi}}\right)+\overline{\boldsymbol{\psi}}
$$

with $\rho$ slightly greater than one (typically 1.01). The purpose is to account for a slight under representation of variance due to the use of a small ensemble.

Bishop et al. (2001) used an implementation of the EnKF in an observation system simulation experiment. Ensemble predicted error statistics were used to determine the optimal configuration of future targeted observations. The application typically looked at a case where additional targeted measurements could be deployed over the next few days and the deployment could be optimized to minimize the forecast errors in a selected region. The methodology was named Ensemble Transform Kalman Filter and it was further examined by Majumdar et al. (2001).

Reichle et al. (2002) give a nice discussion of the EnKF in relation to the optimal representer solution. They find good convergence of the EnKF toward the representer solution with the difference being caused by the Gaussian assumptions used in the EnKF at analysis steps. These are avoided in the representer method which solves for the maximum likelihood smoother estimate.

Evensen (2002) provided an intercomparison and review of sequential assimilation methods including some simple examples.

Bertino et al. (2002) applied the EnKF and the Reduced Rank Square Root (RRSQRT) filter with a model for the Odra estuary. The two methods were compared and used to assimilate real observations to assess the potential for operational forecasting in the lagoon. This is a relatively linear model and the EnKF and the RRSQRT filter provided similar results.

Eknes and Evensen (2002) examined the EnKF with a 1$\mathrm{D}$ three component marine ecosystem model with focus on sensitivity to the characteristics of the assimilated measurements and the ensemble size. It was found that the EnKF could handle strong nonlinearities and instabilities which occur during the spring bloom.

Allen et al. (2002) takes the Eknes and Evensen (2002) work one step further by applying the method with a 1-D version of ERSEM for a site in the Mediterranean Sea. They showed that even with such a complex model it is possible to find an improved estimate by assimilating in situ data into the model.

Haugen and Evensen (2002) applied the EnKF to assimilate sea level anomalies and sea surface temperature data into a version of the Miami Isopycnic Coordinate Ocean Model
(MICOM) by Bleck et al. (1992) for the Indian Ocean. The paper provided an analysis of regionally dependent covariance functions in the tropics and subtropics and also the multivariate impact of assimilating satellite observations.

Brusdal et al. (2002) discussed a similar application as Haugen et al. (2002), but focussed on the North Atlantic. In addition, this paper included an extensive intercomparison of the theoretical background of the EnKF, EnKS and the SEEK filter, and also compared results from these methods using the same model and measurements. This paper, together with Haugen et al. (2002), are the first applications of the EnKF with a full-blown OGCM in a realistic application, and have proved the feasibility of the assimilation system for real oceanographic problems.

Natvik and Evensen (2002a,b) presented the first realistic 3-D application of the EnKF with a marine ecosystem model. These papers proved the feasibility of assimilating SeaWiFS ocean colour data to control the evolution of a marine ecosystem model. In addition several diagnostic methods were introduced which can be used to examine the statistical and other properties of the ensemble.

Mitchell et al. (2002) examined the EnKF with a global atmospheric general circulation model with simulated data resembling realistic operational observations. They assimilated 80000 observations a day. The system was examined with respect to required ensemble size, and the effect of localization (local analysis at a grid point using only nearby measurements). It was found that severe localization could lead to imbalance, but with large enough ratio of influence for the measurements, this was not a problem and no digital filtering was required. In the experiments they also included model errors and demonstrated the importance of this to avoid filter divergence. This work is a significant step forward and it shows promising results with respect to using the EnKF with atmospheric forecast models.

Keppenne and Rienecker (2002) implemented a massively parallel version of the EnKF with the Poseidon isopycnic coordinate ocean model for the tropical Pacific. They demonstrated the assimilation of in situ observations and focussed on the parallelization of the model and analysis scheme for computers with distributed memory. They also showed that regionalization of background covariances has negligible impact on the quality of the analysis.

\subsection{Other ensemble based filters}

The EnKF can also be related to some other sequential filters such as the Singular Evolutive Extended Kalman (SEEK) filter by Pham et al. (1998); Brasseur et al. (1999); Carmillet et al. (2001) (see also Brusdal et al., 2002, for an intercomparison of the SEEK and the EnKF); the Reduced Rank Square Root (RRSQRT) filter by Verlaan and Heemink (2001); and the Error Subspace Statistical Estimation (ESSE) filter by Lermusiaux and Robinson (1999a,b); Lermusiaux (2001) which can be interpreted as an EnKF where the analysis is computed in the space spanned by the EOFs of the ensemble. 
Anderson (2001) proposed a method denoted the "Ensemble Adjustment Kalman Filter" where the analysis is computed without adding perturbations to the observations. This still gives the correct mean of the analyzed ensemble but in the EnKF it would give too low variance as explained by Burgers et al. (1998). This is accounted for by deriving a linear operator which replaces the traditional gain matrix and results in an updated ensemble which is consistent with theory. A drawback may be the required inversion of the measurement error covariance when this is nondiagonal. This method becomes a variant of the square root algorithm used by Bishop et al. (2001). It is demonstrated that for small ensembles (1020 members) the EAKF performs better than the EnKF.

Whitaker and Hamill (2002) proposed another version of the EnKF where the perturbation of observations are avoided. The scheme provides a better estimate of the analysis variance by avoiding the sampling errors of the observation perturbations. The scheme was tested for small ensemble sizes (10-20 members) where it had a clear benefit on the results. The scheme is based on a redefinition of the Kalman gain derived from the equation

$$
\begin{aligned}
\boldsymbol{P}_{\mathrm{e}}^{\mathrm{a}} & =(\boldsymbol{I}-\boldsymbol{K} \boldsymbol{H}) \boldsymbol{P}^{\mathrm{f}}\left(\boldsymbol{I}-\boldsymbol{H}^{\mathrm{T}} \boldsymbol{K}^{\mathrm{T}}\right)+\boldsymbol{K} \boldsymbol{R} \boldsymbol{K}^{\mathrm{T}} \\
& =(\boldsymbol{I}-\boldsymbol{K} \boldsymbol{H}) \boldsymbol{P}^{\mathrm{f}} .
\end{aligned}
$$

where the term $\boldsymbol{K} \boldsymbol{R} \boldsymbol{K}^{\mathrm{T}}=0$ without perturbations of measurements. A solution of this equation is

$$
\begin{aligned}
\boldsymbol{K} & =\boldsymbol{P}^{\mathrm{f}} \boldsymbol{H}^{\mathrm{T}}\left[\left(\sqrt{\boldsymbol{H} \boldsymbol{P}^{\mathrm{f}} \boldsymbol{H}^{\mathrm{T}}+\boldsymbol{R}}\right)^{-1}\right]^{\mathrm{T}} \\
& \times\left[\sqrt{\boldsymbol{H} \boldsymbol{P}^{\mathrm{f}} \boldsymbol{H}^{\mathrm{T}}+\boldsymbol{R}}+\sqrt{\boldsymbol{R}}\right]^{-1} .
\end{aligned}
$$

This is essentially a Monte Carlo implementation of the square root filter and was named (EnSRF).

\subsection{Ensemble smoothers}

Some publications have focussed on the extension of the EnKF to a smoother. The first formulation was given by van Leeuwen and Evensen (1996) who introduced the Ensemble Smoother (ES). This method has later been examined in Evensen (1997) with the Lorenz attractor; applied with a QG model to find a steady mean flow by van Leeuwen (1999b) and for the time dependent problem in van Leeuwen (2001); and for fish stock assessment by Gronnevik and Evensen (2001). Evensen and van Leeuwen (2000) re-examined the smoother formulation and derived a new algorithm with better properties named the Ensemble Kalman Smoother (EnKS). This method has also been examined in Gronnevik and Evensen (2001) and Brusdal et al. (2002).

\subsection{Nonlinear filters and smoothers}

Another extension of the EnKF relates to the derivation of an efficient method for solving the nonlinear filtering problem, i.e., taking non-Gaussian contributions in the predicted error statistics into account when computing the analysis. These are discarded in the EnKF (see Evensen and van Leeuwen, 2000), and a fully nonlinear filter is expected to improve the results when used with nonlinear dynamical models with multimodal behaviour where the predicted error statistics are far from Gaussian. Implementations of nonlinear filters have been proposed by Miller et al. (1999), Anderson and Anderson (1999), Pham (2001) and Miller and Ehret (2002), although they are still not practical for high dimensional systems due to large numerical cost. A promising exception is the approach taken by van Leeuwen (2002) based on importance sampling.

\section{Sequential data assimilation}

This section gives a brief introduction to sequential data assimilation methodologies such as the Kalman Filter (KF) and the Extended Kalman Filter (EKF) and outline the general theory of the EnKF.

\subsection{A variance minimizing analysis}

The Kalman Filter is a sequential filter method, which means that the model is integrated forward in time and whenever measurements are available these are used to reinitialize the model before the integration continues. We neglect the time index and denote a model forecast and analysis as $\boldsymbol{\psi}^{\mathrm{f}}$ and $\boldsymbol{\psi}^{\mathrm{a}}$ respectively and the measurements are contained in $\boldsymbol{d}$. Further, the respective covariances for model forecast, analysis and measurements are denoted $\boldsymbol{P}^{\mathrm{f}}, \boldsymbol{P}^{\mathrm{a}}$ and $\boldsymbol{R}$, and the analysis equation becomes

$$
\boldsymbol{\psi}^{\mathrm{a}}=\boldsymbol{\psi}^{\mathrm{f}}+\boldsymbol{P}^{\mathrm{f}} \boldsymbol{H}^{\mathrm{T}}\left(\boldsymbol{H} \boldsymbol{P}^{\mathrm{f}} \boldsymbol{H}^{\mathrm{T}}+\boldsymbol{R}\right)^{-1}\left(\boldsymbol{d}-\boldsymbol{H} \boldsymbol{\psi}^{\mathrm{f}}\right),
$$

with the analysis error covariances given as

$$
\boldsymbol{P}^{\mathrm{a}}=\boldsymbol{P}^{\mathrm{f}}-\boldsymbol{P}^{\mathrm{f}} \boldsymbol{H}^{\mathrm{T}}\left(\boldsymbol{H} \boldsymbol{P}^{\mathrm{f}} \boldsymbol{H}^{\mathrm{T}}+\boldsymbol{R}\right)^{-1} \boldsymbol{H} \boldsymbol{P}^{\mathrm{f}} .
$$

Here $\boldsymbol{H}$ is the measurement operator relating the true model state $\boldsymbol{\psi}^{\mathrm{t}}$ to the observations $\boldsymbol{d}$ allowing for measurement errors $\epsilon$, i.e.

$$
\boldsymbol{d}=\boldsymbol{H} \boldsymbol{\psi}^{\mathrm{t}}+\boldsymbol{\epsilon}
$$

The reinitialization, $\boldsymbol{\psi}^{\mathrm{a}}$, is determined as a weighted linear combination of the model prediction, $\boldsymbol{\psi}^{\mathrm{f}}$, and the measurements, $\boldsymbol{d}$. The weights are the inverses of the error covariances for the model prediction and the measurements, and the optimal linear-combination becomes the Best Linear Unbiased Estimator (BLUE).

The error covariances for the measurements, $\boldsymbol{R}$, need to be prescribed based on our best knowledge about their accuracy and the methodologies used to collect them. The error covariances for the model prediction is computed by solving an equation for the time evolution of the error covariance matrix of the model state.

A derivation of these equations can be found in several publications (see e.g. Burgers et al., 1998). 


\subsection{The Kalman Filter}

Given a linear dynamical model written on discrete form as

$$
\boldsymbol{\psi}_{k+1}=\boldsymbol{F} \boldsymbol{\psi}_{k}
$$

the error covariance equation becomes

$$
\boldsymbol{P}_{k+1}=\boldsymbol{F} \boldsymbol{P}_{k} \boldsymbol{F}^{\mathrm{T}}+\boldsymbol{Q},
$$

where the matrix $Q$ is the error covariance matrix for the model errors. The model is assumed to contain errors, e.g. due to neglected physics and numerical approximations. The equations (7) and (8) are integrated to produce the forecasts $\boldsymbol{\psi}^{\mathrm{f}}$ and $\boldsymbol{P}^{\mathrm{f}}$, used in the analysis equations (4) and (5).

\subsection{The Extended Kalman Filter}

With a nonlinear model

$$
\boldsymbol{\psi}_{k+1}=\boldsymbol{f}\left(\boldsymbol{\psi}_{k}\right)
$$

the error covariance equation would still be (8) but with $\boldsymbol{F}$ being the tangent linear operator (Jacobian) of $\boldsymbol{f}(\boldsymbol{\psi})$. Thus, a linearized and approximate equation is used for the prediction of error statistics in the Extended Kalman Filter (EKF). A comprehensive discussion of the properties of the EKF can be found in the literature, but for a convenient summary which intercompares the EKF with Ensemble Kalman Filter (EnKF), to be discussed next, see Evensen (2002).

\subsection{The Ensemble Kalman Filter}

The ensemble Kalman filter as proposed by Evensen (1994b) and later clearified by Burgers et al. (1998) is now introduced. We will adapt a three stage presentation starting with the representation of error statistics using an ensemble of model states, then an alternative to the traditional error covariance equation is proposed for the prediction of error statistics, and finally a consistent analysis scheme is presented.

3.4.1 Representation of error statistics The error covariance matrices for the forecasted and the analyzed estimate, $\boldsymbol{P}^{\mathrm{f}}$ and $\boldsymbol{P}^{\mathrm{a}}$, are in the Kalman filter defined in terms of the true state as

$$
\begin{aligned}
& \boldsymbol{P}^{\mathrm{f}}=\overline{\left(\boldsymbol{\psi}^{\mathrm{f}}-\boldsymbol{\psi}^{\mathrm{t}}\right)\left(\boldsymbol{\psi}^{\mathrm{f}}-\boldsymbol{\psi}^{\mathrm{t}}\right)^{\mathrm{T}}}, \\
& \boldsymbol{P}^{\mathrm{a}}=\overline{\left(\boldsymbol{\psi}^{\mathrm{a}}-\boldsymbol{\psi}^{\mathrm{t}}\right)\left(\boldsymbol{\psi}^{\mathrm{a}}-\boldsymbol{\psi}^{\mathrm{t}}\right)^{\mathrm{T}}},
\end{aligned}
$$

where the overline denotes an expectation value, $\boldsymbol{\psi}$ is the model state vector at a particular time and the superscripts $\mathrm{f}$, $\mathrm{a}$, and $\mathrm{t}$ represent forecast, analyzed, and true state, respectively. However, since the true state is not known, it is more convenient to consider ensemble covariance matrices around the ensemble mean, $\bar{\psi}$,

$$
\begin{aligned}
& \boldsymbol{P}^{\mathrm{f}} \simeq \boldsymbol{P}_{\mathrm{e}}^{\mathrm{f}}=\overline{\left(\boldsymbol{\psi}^{\mathrm{f}}-\overline{\boldsymbol{\psi}^{\mathrm{f}}}\right)\left(\boldsymbol{\psi}^{\mathrm{f}}-\overline{\boldsymbol{\psi}^{\mathrm{f}}}\right)^{\mathrm{T}}}, \\
& \boldsymbol{P}^{\mathrm{a}} \simeq \boldsymbol{P}_{\mathrm{e}}^{\mathrm{a}}=\overline{\left(\boldsymbol{\psi}^{\mathrm{a}}-\overline{\boldsymbol{\psi}^{\mathrm{a}}}\right)\left(\boldsymbol{\psi}^{\mathrm{a}}-\overline{\boldsymbol{\psi}^{\mathrm{a}}}\right)^{\mathrm{T}}},
\end{aligned}
$$

where now the overline denote an average over the ensemble. Thus, we can use an interpretation where the ensemble mean is the best estimate and the spreading of the ensemble around the mean is a natural definition of the error in the ensemble mean.

Since the error covariances as defined in (12) and (13) are defined as ensemble averages, there will clearly exist infinitively many ensembles with an error covariance equal to $\boldsymbol{P}_{\mathrm{e}}^{\mathrm{f}}$ and $\boldsymbol{P}_{\mathrm{e}}^{\mathrm{a}}$. Thus, instead of storing a full covariance matrix, we can represent the same error statistics using an appropriate ensemble of model states. Given an error covariance matrix, an ensemble of finite size will always provide an approximation to the error covariance matrix. However, when the size of the ensemble $N$ increases the errors in the representation will decrease proportional to $1 / \sqrt{N}$.

Suppose now that we have $N$ model states in the ensemble, each of dimension $n$. Each of these model states can be represented as a single point in an $n$-dimensional state space. All the ensemble members together will constitute a cloud of points in the state space. Such a cloud of points in the state space can be approximately described using a probability density function

$$
\phi(\boldsymbol{\psi})=\frac{d N}{N},
$$

where $d N$ is the number of points in a small unit volume and $N$ is the total number of points. With knowledge about either $\phi$ or the ensemble representing $\phi$ we can calculate whichever statistical moments (such as mean, covariances etc.) we want whenever they are needed.

The conclusion so far is that the information contained by a full probability density function can be represented by an ensemble of model states.

3.4.2 Prediction of error statistics The EnKF was designed to resolve two major problems related to the use of the EKF with nonlinear dynamics in large state spaces. The EKF applies a closure scheme where third- and higher order moments in the error covariance equation are discarded. This linearization has been shown to be invalid in a number of applications, e.g., Evensen (1992) and Miller et al. (1994). In fact, the equation is no longer the fundamental equation for the error evolution when the dynamical model is nonlinear. In Evensen (1994b) it was shown that a Monte Carlo method can be used to solve an equation for the time evolution of the probability density of the model state, as an alternative to using the approximate error covariance equation in the EKF.

For a nonlinear model where we appreciate that the model is not perfect and contains model errors, we can write it as a stochastic differential equation (on continuous form) as

$$
d \boldsymbol{\psi}=\boldsymbol{f}(\boldsymbol{\psi}) d t+\boldsymbol{g}(\boldsymbol{\psi}, d \boldsymbol{q}) .
$$

This equation states that an increment in time will yield an increment in $\psi$, which in addition, is influenced by a random contribution from the stochastic forcing term, $\boldsymbol{g}(\boldsymbol{\psi}, d \boldsymbol{q})$, representing the model errors. The $d \boldsymbol{q}$ describe a vector Brownian motion process with covariance $Q d t$. Because the model 
is nonlinear, $\boldsymbol{g}$ is not an explicit function of the random variable $d \boldsymbol{q}$ so the Ito interpretation of the stochastic differential equation has to be used instead of the Statonovitz interpretation Jazwinski (1970).

When the model errors are additive, i.e., when the stochastic forcing term can be written as $\boldsymbol{g}(\boldsymbol{\psi}, d \boldsymbol{q})=g(\boldsymbol{\psi}) d \boldsymbol{q}$, one can derive the Fokker-Planck equation (also named Kolmogoro equation) which describes the time evolution of the probability density $\phi(\boldsymbol{\psi})$ of the model state,

$$
\frac{\partial \phi}{\partial t}+\sum_{i} \frac{\partial\left(f_{i} \phi\right)}{\partial \psi_{i}}=\frac{1}{2} \sum_{i, j} \frac{\partial^{2} \phi\left(\boldsymbol{g} \boldsymbol{Q} \boldsymbol{g}^{T}\right)_{i j}}{\partial \psi_{i} \partial \psi_{j}}
$$

where $f_{i}$ is the component number $i$ of the model operator $\boldsymbol{f}$ and $\boldsymbol{g} \boldsymbol{Q} \boldsymbol{g}^{\mathrm{T}}$ is the covariance matrix for the model errors.

This equation does not apply any important approximations and can be considered as the fundamental equation for the time evolution of error statistics. A detailed derivation is given in Jazwinski (1970). The equation describes the change of the probability density in a local "volume" which is dependent on the divergence term describing a probability flux into the local "volume" (impact of the dynamical equation) and the diffusion term which tends to flatten the probability density due to the effect of stochastic model errors. If (16) could be solved for the probability density function, it would be possible to calculate statistical moments like the mean state and the error covariance for the model forecast to be used in the analysis scheme.

The EnKF applies a socalled Markov Chain Monte Carlo (MCMC) method to solve (16). The probability density can be represented using a large ensemble of model states and by integrating these model states forward in time according to the model dynamics described by the stochastic differential equation (15), this ensemble prediction is equivalent to solving the Fokker Planck equation using a MCMC method. This procedure forms the backbone for the EnKF.

A linear model for a Gauss-Markov process in which the initial condition is assumed to be taken from a normal distribution will have a probability density which is completely characterized by its mean and covariance matrix for all times. One can then derive exact equations for the evolution of the mean and the covariance matrix as a simpler alternative than solving the full Kolmogorov's equation. Such moments of Kolmogorov's equation, including the error covariance equation (8), are easy to derive, and several methods are illustrated by Jazwinski (1970, examples 4.19-4.21).

For a nonlinear model, the mean and covariance matrix will not in general characterize $\phi(\boldsymbol{\psi}, t)$. They do, however, determine the mean path and the dispersion about that path, and it is possible to solve approximate equations for the moments, which is the procedure characterizing the extended Kalman filter.

An alternative to the approximate stochastic dynamic approach for solving Kolmogorov's equation and predicting the error statistics is to use Monte Carlo methods. A large cloud of model states (points in state space) can be used to represent a specific probability density function. By integrating such an ensemble of states forward in time, it is easy to calculate approximate estimates for moments of the probability density function at different time levels. In this context the Monte Carlo method might be considered a particle method in the state space.

's 3.4.3 An analysis scheme The KF analysis scheme was based on the definitions of $\boldsymbol{P}^{\mathrm{f}}$ and $\boldsymbol{P}^{\mathrm{a}}$ as given by equations (10) and (11). We will now give a derivation of the analysis scheme where the ensemble covariances are used as defined by (12) and (13). This is convenient since in practical implementations one is doing exactly this, and it will also lead to a consistent formulation of the EnKF.

As will be shown later it is essential that the observations are treated as random variables having a distribution with mean equal to the first guess observations and covariance equal to $\boldsymbol{R}$. Thus, we start by defining an ensemble of observations

$$
\boldsymbol{d}_{j}=\boldsymbol{d}+\boldsymbol{\epsilon}_{j}
$$

where $j$ counts from 1 to the number of model state ensemble members $N$. Next we define the ensemble covariance matrix of the measurements as

$$
\boldsymbol{R}_{\mathrm{e}}=\overline{\boldsymbol{\epsilon} \boldsymbol{\epsilon}^{\mathrm{T}}}
$$

and, of course, in the limit of an infinite ensemble this matrix will converge toward the prescribed error covariance matrix $\boldsymbol{R}$ used in the standard Kalman filter.

The analysis step for the EnKF consists of the following updates performed on each of the model state ensemble members

$$
\boldsymbol{\psi}_{j}^{\mathrm{a}}=\boldsymbol{\psi}_{j}^{\mathrm{f}}+\boldsymbol{P}_{\mathrm{e}}^{\mathrm{f}} \boldsymbol{H}^{\mathrm{T}}\left(\boldsymbol{H} \boldsymbol{P}_{\mathrm{e}}^{\mathrm{f}} \boldsymbol{H}^{\mathrm{T}}+\boldsymbol{R}_{\mathrm{e}}\right)^{-1}\left(\boldsymbol{d}_{j}-\boldsymbol{H} \boldsymbol{\psi}_{j}^{\mathrm{f}}\right) .
$$

Note that equation (19) implies that

$$
\overline{\boldsymbol{\psi}^{\mathrm{a}}}=\overline{\boldsymbol{\psi}^{\mathrm{f}}}+\boldsymbol{P}_{\mathrm{e}}^{\mathrm{f}} \boldsymbol{H}^{\mathrm{T}}\left(\boldsymbol{H} \boldsymbol{P}_{\mathrm{e}}^{\mathrm{f}} \boldsymbol{H}^{\mathrm{T}}+\boldsymbol{R}_{\mathrm{e}}\right)^{-1}\left(\overline{\boldsymbol{d}}-\boldsymbol{H} \overline{\boldsymbol{\psi}^{\mathrm{f}}}\right),
$$

where $\overline{\boldsymbol{d}}=\boldsymbol{d}$ is the first guess vector of measurements. Thus, the relation between the analyzed and forecasted ensemble mean is identical to the relation between the analyzed and forecasted state in the standard Kalman filter in equation (4), apart from the use of $\boldsymbol{P}_{\mathrm{e}}^{\mathrm{f}, \mathrm{a}}$ and $\boldsymbol{R}_{\mathrm{e}}$ instead of $\boldsymbol{P}^{\mathrm{f}, \mathrm{a}}$ and $\boldsymbol{R}$. Note that the introduction of an ensemble of observations does not make any difference for the update of the ensemble mean since this does not affect equation (20).

If the mean, $\overline{\psi^{\mathrm{a}}}$, is considered to be the best estimate, then the linearity of the analysis scheme makes it an arbitrary choice whether one updates the mean using the first guess observations $\boldsymbol{d}$, or if one updates each of the ensemble members using the perturbed observations (17). However, it will now be shown that by updating each of the ensemble members using the perturbed observations one also creates a new ensemble having the correct error statistics for the analysis. The updated ensemble can then be integrated forward in time till the next observation time.

Moreover, the error covariance, $\boldsymbol{P}_{\mathrm{e}}^{\mathrm{a}}$, of the analyzed ensemble is reduced in the same way as in the standard Kalman 
Filter. We now derive the analyzed error covariance estimate resulting from the analysis scheme given above, but using the standard Kalman filter form for the analysis equations. First, note that equations (19) and (20) are used to get

$$
\boldsymbol{\psi}_{j}^{\mathrm{a}}-\overline{\boldsymbol{\psi}^{\mathrm{a}}}=\left(\boldsymbol{I}-\boldsymbol{K}_{\mathrm{e}} \boldsymbol{H}\right)\left(\boldsymbol{\psi}_{j}^{\mathrm{f}}-\overline{\boldsymbol{\psi}^{\mathrm{f}}}\right)+\boldsymbol{K}_{\mathrm{e}}\left(\boldsymbol{d}_{j}-\overline{\boldsymbol{d}}\right),
$$

where we have used the definition of the Kalman gain,

$$
\boldsymbol{K}_{\mathrm{e}}=\boldsymbol{P}_{\mathrm{e}}^{\mathrm{f}} \boldsymbol{H}^{\mathrm{T}}\left(\boldsymbol{H} \boldsymbol{P}_{\mathrm{e}}^{\mathrm{f}} \boldsymbol{H}^{\mathrm{T}}+\boldsymbol{R}_{\mathrm{e}}\right)^{-1} .
$$

The derivation is then as follows,

$$
\begin{aligned}
\boldsymbol{P}_{\mathrm{e}}^{\mathrm{a}}= & \overline{\left(\boldsymbol{\psi}^{\mathrm{a}}-\overline{\boldsymbol{\psi}^{\mathrm{a}}}\right)\left(\boldsymbol{\psi}^{\mathrm{a}}-\overline{\boldsymbol{\psi}^{\mathrm{a}}}\right)^{\mathrm{T}}} \\
= & \left(\boldsymbol{I}-\boldsymbol{K}_{\mathrm{e}} \boldsymbol{H}\right) \boldsymbol{P}_{\mathrm{e}}^{\mathrm{f}}\left(\boldsymbol{I}-\boldsymbol{H}^{\mathrm{T}} \boldsymbol{K}_{\mathrm{e}}^{\mathrm{T}}\right)+\boldsymbol{K}_{\mathrm{e}} \boldsymbol{R}_{\mathrm{e}} \boldsymbol{K}_{\mathrm{e}}^{\mathrm{T}} \\
= & \boldsymbol{P}_{\mathrm{e}}^{\mathrm{f}}-\boldsymbol{K}_{\mathrm{e}} \boldsymbol{H} \boldsymbol{P}_{\mathrm{e}}^{\mathrm{f}}-\boldsymbol{P}_{\mathrm{e}}^{\mathrm{f}} \boldsymbol{H}^{\mathrm{T}} \boldsymbol{K}_{\mathrm{e}}^{\mathrm{T}} \\
& \quad \quad \quad \boldsymbol{K}_{\mathrm{e}}\left(\boldsymbol{H} \boldsymbol{P}_{\mathrm{e}}^{\mathrm{f}} \boldsymbol{H}^{\mathrm{T}}+\boldsymbol{R}_{\mathrm{e}}\right) \boldsymbol{K}_{\mathrm{e}}^{\mathrm{T}} \\
= & \left(\boldsymbol{I}-\boldsymbol{K}_{\mathrm{e}} \boldsymbol{H}\right) \boldsymbol{P}_{\mathrm{e}}^{\mathrm{f}} .
\end{aligned}
$$

Note that this derivation clearly states that the observations $\boldsymbol{d}$ must be treated as random variables to get the measurement error covariance matrix $\boldsymbol{R}_{\mathrm{e}}$ into the expression.

3.4.4 Summary We now have a complete system of equations which constitutes the ensemble Kalman filter (EnKF), and the resemblance with the standard Kalman filter is maintained. This is also true for the forecast step. Each ensemble member evolves in time according to the model dynamics. The ensemble covariance matrix of the errors in the model equations, given by

$$
\boldsymbol{Q}_{\mathrm{e}}=\overline{d \boldsymbol{q}_{k} d \boldsymbol{q}_{k}^{\mathrm{T}}},
$$

converges to $Q$ in the limit of infinite ensemble size. The ensemble mean then evolves according to the equation

$$
\begin{aligned}
\overline{\boldsymbol{\psi}_{k+1}} & =\overline{\boldsymbol{f}\left(\boldsymbol{\psi}_{k}\right)} \\
& =\boldsymbol{f}\left(\overline{\boldsymbol{\psi}_{k}}\right)+\text { n.l. }
\end{aligned}
$$

where n.l. represents the terms which may arise if $f$ is nonlinear. One of the advantages of the EnKF is that the effect of these terms is retained since each ensemble member is integrated independently by the model.

The error covariance of the ensemble evolves according to

$$
\boldsymbol{P}_{\mathrm{e}}^{k+1}=\boldsymbol{F} \boldsymbol{P}_{\mathrm{e}}^{k} \boldsymbol{F}^{\mathrm{T}}+\boldsymbol{Q}_{\mathrm{e}}+\text { n.l., }
$$

where $\boldsymbol{F}$ is the tangent linear operator evaluated at the current time step. This is again an equation of the same form as is used in the standard Kalman filter, except of the extra terms n.l. that may appear if $\boldsymbol{f}$ is non-linear. Implicitly, the EnKF retains these terms also for the error covariance evolution.

Thus, if the ensemble mean is used as the best estimate, with the ensemble covariance $\boldsymbol{P}_{\mathrm{e}}$ interpreted as the error covariance $\boldsymbol{P}$, and by defining the observation error covariance matrix $\boldsymbol{R}_{\mathrm{e}}=\boldsymbol{R}$ and the model error covariance $\boldsymbol{Q}_{\mathrm{e}}=\boldsymbol{Q}$, the EnKF and the standard Kalman filter become identical. This discussion shows that there is a unique correspondence between the EnKF and the standard Kalman filter (for linear dynamics), and that one can certainly interpret the ensemble covariances as error covariances while the ensemble mean is used as the best guess trajectory.

For nonlinear dynamics the so called extended Kalman filter may be used and is given by the evolution equations (25) and (26) with the n.l. terms neglected. The ensemble Kalman filter includes the full effect of these terms and there are no linearizations or closure assumptions applied. In addition, there is no need for a tangent linear operator, such as $\boldsymbol{F}$, or its adjoint, and this makes the EnKF very easy to implement for practical applications.

This leads to an interpretation of the EnKF as a purely statistical Monte Carlo method where the ensemble of model states evolves in state space with the mean as the best estimate and the spreading of the ensemble as the error variance. At measurement times each observation is represented by another ensemble, where the mean is the actual measurement and the variance of the ensemble represents the measurement errors.

\section{Practical formulation and interpretation}

This section discusses the EnKF in more detail with focus on the practical formulation and interpretation. It is shown that an interpretation in the "ensemble space" provides a better understanding of the actual algorithm and also allows for very efficient algorithms to be developed.

\subsection{The initial ensemble}

The initial ensemble should ideally be chosen to properly represent the error statistics of the initial guess for the model state. However, a modest mis-specification of the initial ensemble does normally not influence the results very much over time. The rule of thumb seems to be that one needs to create an ensemble of model states by adding some kind of perturbations to a best guess estimate, and then integrate the ensemble over a time interval covering a few characteristic time scales of the dynamical system. This will ensure that the system is in dynamical balance and that proper multivariate correlations have developed.

The perturbations can be created in different ways. The simplest is to sample random numbers (for a scalar model), random curves (for a 1-D model) or random fields (for a model with 2 or higher dimensions), from a specified distribution. In Appendix E there is an example of a procedure for generating such random perturbations.

\subsection{The ensemble integration}

The ensemble of model states is integrated forward in time according to the stochastic equation (15). In a practical implementation this becomes just a standard integration of the 
numerical model but subject to a stochastic noise which resembles the uncertainties in the model. Note that the EnKF allows for a wide range of noise models. Stochastic terms can be added to all poorly known model parameters and one is not restricted to use Gaussian distributed noise. Further, it is possible to use time correlated (red) noise.

4.2.1 Simulation of model errors The following equation can be used for simulating the time evolution of model errors:

$$
\boldsymbol{q}_{k}=\alpha \boldsymbol{q}_{k-1}+\sqrt{1-\alpha^{2}} \boldsymbol{w}_{k-1} .
$$

Here we assume that $\boldsymbol{w}_{k}$ is a sequence of white noise drawn from a distribution of smooth pseudo random fields with mean equal to zero and variance equal to one. Such fields can be generated using the algorithm presented in the Appendix E. The coefficient $\alpha \in[0,1)$ determines the time decorrelation of the stochastic forcing, e.g., $\alpha=0$ generates a sequence which is white in time, while $\alpha=1$ will remove the stochastic forcing and represent the model errors with a random field which is constant in time.

This equation ensures that the variance of $\boldsymbol{q}_{k}$ is equal to one as long as the variance of $\boldsymbol{q}_{k-1}$ is one. Thus, this equation will produce a sequence of time correlated pseudo random fields with mean equal to zero and variance equal to one.

The covariance in time between $\boldsymbol{q}_{i}$ and $\boldsymbol{q}_{j}$, determined by equation (27), is

$$
\overline{\boldsymbol{q}_{i} \boldsymbol{q}_{j}}=\alpha^{|i-j|} .
$$

Determination of $\alpha$. The factor $\alpha$ should be related to the time step used and a specified time decorrelation length $\tau$. The equation (27), when excluding the stochastic term, resembles a difference approximation to

$$
\frac{\partial q}{\partial t}=-\frac{1}{\tau} q
$$

which states that $q$ is damped with a ratio $e^{-1}$ over a time period $t=\tau$. A numerical approximation becomes

$$
q_{k}=\left(1-\frac{\Delta t}{\tau}\right) q_{k-1},
$$

where $\Delta t$ is the time step. Thus, we 'define $\alpha$ as

$$
\alpha=1-\frac{\Delta t}{\tau},
$$

where $\tau \geq \Delta t$.

Physical model. Based on random walk theory (see below), the physical model can be written as

$$
\boldsymbol{\psi}_{k}=\boldsymbol{f}\left(\boldsymbol{\psi}_{k-1}\right)+\sqrt{\Delta t} \sigma \rho \boldsymbol{q}_{k},
$$

where $\sigma$ is the standard deviation of the model error and $\rho$ is a factor to be determined. The choice of the stochastic term is explained next.
Variance growth due to the stochastic forcing. To explain the choice of the stochastic term in Equation (32) we will use a simple random walk model for illustration, i.e.,

$$
\boldsymbol{\psi}_{k}=\boldsymbol{\psi}_{k-1}+\sqrt{\Delta t} \sigma \rho \boldsymbol{q}_{k}
$$

This equation can be rewritten as

$$
\boldsymbol{\psi}_{k}=\boldsymbol{\psi}_{0}+\sqrt{\Delta t} \sigma \rho \sum_{i=0}^{k-1} \boldsymbol{q}_{i+1} .
$$

The variance can be found by squaring (34) and taking the ensemble average, i.e.,

$$
\begin{aligned}
\overline{\boldsymbol{\psi}_{n} \boldsymbol{\psi}_{n}^{\mathrm{T}}} & =\overline{\boldsymbol{\psi}_{0} \boldsymbol{\psi}_{0}^{\mathrm{T}}}+\Delta t \sigma^{2} \rho^{2} \overline{\left(\sum_{k=0}^{n-1} \boldsymbol{q}_{k+1}\right)\left(\sum_{k=0}^{n-1} \boldsymbol{q}_{k+1}\right)^{\mathrm{T}}} \\
& =\overline{\boldsymbol{\psi}_{0} \boldsymbol{\psi}_{0}^{\mathrm{T}}}+\Delta t \sigma^{2} \sum_{j=0}^{n-1} \sum_{i=0}^{n-1} \overline{\boldsymbol{q}_{i+1} \boldsymbol{q}_{j+1}^{\mathrm{T}}} \\
& =\overline{\boldsymbol{\psi}_{0} \boldsymbol{\psi}_{0}^{\mathrm{T}}}+\Delta t \sigma^{2} \rho^{2} \sum_{j=0}^{n-1} \sum_{i=0}^{n-1} \alpha^{|i-j|} \\
& =\overline{\boldsymbol{\psi}_{0} \boldsymbol{\psi}_{0}^{\mathrm{T}}}+\Delta t \sigma^{2} \rho^{2}\left(-n+2 \sum_{i=0}^{n-1}(n-i) \alpha^{i}\right) \\
& =\overline{\boldsymbol{\psi}_{0} \boldsymbol{\psi}_{0}^{\mathrm{T}}}+\Delta t \sigma^{2} \rho^{2} \frac{n-2 \alpha+n \alpha^{2}+2 n \alpha^{n+1}}{(1-\alpha)^{2}}
\end{aligned}
$$

where the expression (28) has been used. The double sum in equation (37) is just summing elements in a matrix and is replaced by a single sum operating on diagonals of constant values. The summation in (38) has an explicit solution (Gradshteyn and Ryzhik, 1979, formula 0.113).

If the sequence of model noise $\boldsymbol{q}_{k}$ is white in time ( $\alpha=$ $0)$, this equation implies an increase in variance equal to $\sigma^{2} \rho^{2}$ when equation (33) is iterated $n$ time steps of length $\Delta t$, over one time unit $(n \Delta t=1)$. Thus, in this case $\rho=1$ is a natural choice since this leads to the correct increase in ensemble variance given by $\sigma^{2}$.

In the case with red model errors the increase in ensemble variance over one time unit will increase up to a maximum of $\sigma^{2} \rho^{2} / \Delta t$ in the case when $\alpha=1$ (not covered by the formula 37).

The two equations (27) and (32) provides the standard framework for introducing stochastic model errors when using the EnKF. The formula (39) provides the mean for scaling the perturbations in (32) when changing $\alpha$ and/or the number of time steps per time unit, $n$, to ensure that the ensemble variance growth over a time unit remains the same.

Thus, the constraint that

$$
1=\rho^{2} \Delta t \frac{n-2 \alpha+n \alpha^{2}+2 n \alpha^{n+1}}{(1-\alpha)^{2}},
$$

defines the factor

$$
\rho^{2}=\frac{1}{\Delta t} \frac{(1-\alpha)^{2}}{n-2 \alpha+n \alpha^{2}+2 n \alpha^{n+1}},
$$


whic ensures that the variance growth over time becomes independent of $\alpha$ and $\Delta t$ (as long as the dynamical model is linear).

4.2.2 Estimation of model errors When red model noise is used, correlations will develop between the red noise and the model variables. Thus, during the analysis it is also possible to consistently update the model noise as well as the model state. This was illustrated in an example by Reichle et al. (2002). We introduce a new state vector which consists of $\boldsymbol{\psi}$ augmented with $\boldsymbol{q}$. The two equations (27) and (32) can then be written as

$$
\begin{aligned}
\left(\begin{array}{c}
\boldsymbol{q}_{k} \\
\boldsymbol{\psi}_{k}
\end{array}\right) & =\left(\begin{array}{c}
\alpha \boldsymbol{q}_{k-1} \\
\boldsymbol{f}\left(\boldsymbol{\psi}_{k-1}\right)+\sqrt{\Delta t} \sigma \rho \boldsymbol{q}_{k}
\end{array}\right) \\
& +\left(\begin{array}{c}
\sqrt{1-\alpha^{2}} \boldsymbol{w}_{k-1} \\
0
\end{array}\right) .
\end{aligned}
$$

During the analysis we can now compute covariances between the observed model variable and the model noise vector $\boldsymbol{q}$ and update this together with the state vector. This will lead to a correction of the mean of $\boldsymbol{q}$ as well as a reduction of the variance in the model noise ensemble. Note that this procedure estimates the actual error in the model for each ensemble member, given the prescribed model error statistics.

The form of equation (27) ensures that, over time, $\boldsymbol{q}_{k}$ will approach a distribution with mean equal to zero and variance equal to one, as long as we don't update $\boldsymbol{q}_{k}$ in the analysis scheme.

\subsection{The EnKF analysis scheme}

This section attempts to explain in some detail how the EnKF analysis can be computed efficiently for practical applications. In particular it discusses how the filter can be used to compute a global analysis to an affordable cost, even with a very large number of measurements. It presents a storage scheme which requires only one copy of the ensemble to be kept in memory, and an efficient algorithm for computation of the expensive final matrix multiplication. The concept of a local analysis is discussed in Section 4.4, A discussion is also given on the assimilation of nonlinear measurements in Section 4.5, a problem which is solved by augmenting the model state with the model's measurement equivalents. Moreover, this algorithm also allows for the efficient assimilation of in situ measurements in a consistent manner where one entirely relies on the ensemble predicted error statistics (see Appendix C). Finally a discussion is given on the assimilation of non-synoptic measurements in Section 4.6.

4.3.1 Definitions and the analysis equation Define the matrix holding the ensemble members $\psi_{i} \in \Re^{n}$,

$$
\boldsymbol{A}=\left(\boldsymbol{\psi}_{1}, \boldsymbol{\psi}_{2}, \ldots, \boldsymbol{\psi}_{N}\right) \in \Re^{n \times N},
$$

where $N$ is the number of ensemble members and $n$ is the size of the model state vector.
The ensemble mean is stored in each column of $\overline{\boldsymbol{A}}$ which can be defined as

$$
\overline{\boldsymbol{A}}=\boldsymbol{A} \mathbf{1}_{N},
$$

where $\mathbf{1}_{N} \in \Re^{N \times N}$ is the matrix where each element is equal to $1 / N$. We can then define the ensemble perturbation matrix as

$$
\boldsymbol{A}^{\prime}=\boldsymbol{A}-\overline{\boldsymbol{A}}=\boldsymbol{A}\left(\boldsymbol{I}-\mathbf{1}_{N}\right)
$$

The ensemble covariance matrix $\boldsymbol{P}_{\mathrm{e}} \in \Re^{n \times n}$ can be defined as

$$
\boldsymbol{P}_{\mathrm{e}}=\frac{\boldsymbol{A}^{\prime}\left(\boldsymbol{A}^{\prime}\right)^{\mathrm{T}}}{N-1} .
$$

Given a vector of measurements $\boldsymbol{d} \in \Re^{m}$, with $m$ being the number of measurements, we can define the $N$ vectors of perturbed observations as

$$
\boldsymbol{d}_{j}=\boldsymbol{d}+\boldsymbol{\epsilon}_{j}, \quad j=1, \ldots, N
$$

which can be stored in the columns of a matrix

$$
\boldsymbol{D}=\left(\boldsymbol{d}_{1}, \boldsymbol{d}_{2}, \ldots, \boldsymbol{d}_{N}\right) \in \Re^{m \times N},
$$

while the perturbations can be stored in the matrix

$$
\boldsymbol{\Upsilon}=\left(\boldsymbol{\epsilon}_{1}, \boldsymbol{\epsilon}_{2}, \ldots, \boldsymbol{\epsilon}_{N}\right) \in \Re^{m \times N}
$$

from which we can construct the ensemble representation of the measurement error covariance matrix

$$
\boldsymbol{R}_{\mathrm{e}}=\frac{\boldsymbol{r} \boldsymbol{r}^{\mathrm{T}}}{N-1} .
$$

The standard analysis equation, expressed in terms of the ensemble covariance matrices, is

$$
\boldsymbol{A}^{\mathrm{a}}=\boldsymbol{A}+\boldsymbol{P}_{\mathrm{e}} \boldsymbol{H}^{\mathrm{T}}\left(\boldsymbol{H} \boldsymbol{P}_{\mathrm{e}} \boldsymbol{H}^{\mathrm{T}}+\boldsymbol{R}_{\mathrm{e}}\right)^{-1}(\boldsymbol{D}-\boldsymbol{H} \boldsymbol{A}) .
$$

Using the ensemble of innovation vectors defined as

$$
D^{\prime}=\boldsymbol{D}-\boldsymbol{H} \boldsymbol{A}
$$

and the definitions of the ensemble error covariance matrices in Equations (50) and (46) the analysis can be expressed as

$$
\boldsymbol{A}^{\mathrm{a}}=\boldsymbol{A}+\boldsymbol{A}^{\prime} \boldsymbol{A}^{\prime \mathrm{T}} \boldsymbol{H}^{\mathrm{T}}\left(\boldsymbol{H} \boldsymbol{A}^{\prime} \boldsymbol{A}^{\prime \mathrm{T}} \boldsymbol{H}^{\mathrm{T}}+\boldsymbol{r} \boldsymbol{r}^{\mathrm{T}}\right)^{-1} \boldsymbol{D}^{\prime}
$$

4.3.2 Practical formulation and implementation The traditional way of solving the analysis equation (53) would involve the computation of the eigenvalue decomposition directly from the $m \times m$ matrix,

$$
\boldsymbol{H} \boldsymbol{A}^{\prime} \boldsymbol{A}^{\prime \mathrm{T}} \boldsymbol{H}^{\mathrm{T}}+\boldsymbol{r} \boldsymbol{\Upsilon}^{\mathrm{T}}=\boldsymbol{Z} \boldsymbol{\Lambda} \boldsymbol{Z}^{\mathrm{T}},
$$

which has the inverse

$$
\left(\boldsymbol{H} \boldsymbol{A}^{\prime} \boldsymbol{A}^{\prime \mathrm{T}} \boldsymbol{H}^{\mathrm{T}}+\boldsymbol{r} \boldsymbol{\Upsilon}^{\mathrm{T}}\right)^{-1}=\boldsymbol{Z} \boldsymbol{\Lambda}^{-1} \boldsymbol{Z}^{\mathrm{T}} .
$$

The cost of the eigenvalue decomposition is proportional to $m^{2}$ and becomes un-affordable for large $m$. Note, however that the rank of $\boldsymbol{Z} \boldsymbol{\Lambda} \boldsymbol{Z}^{\mathrm{T}}$ is less than or equal to $N$. Thus, $\boldsymbol{\Lambda}$ 
will have $N$ or less non-zero eigenvalues and it may therefore be possible to use a more efficient eigenvalue decomposition algorithm which only computes and stores the first $N$ columns of $\boldsymbol{Z}$.

It is important to note that if different measurement types are assimilated simultaneously, the model observed model variables need to be made non-dimensional or scaled to have similar variability. This is required to ensure that the eigenvalues to each of the measurement types have the same magnitude. The standard approach for resolving this is to assimilate different measurement types, which normally have uncorrelated errors, sequentially one data set at the time. The validity of this approach has been shown, e.g. by Evensen and van Leeuwen (2000).

Alternative solution for large $m$. If the perturbations used for measurements are chosen such that

$$
\boldsymbol{H} \boldsymbol{A}^{\prime} \boldsymbol{\Upsilon}^{\mathrm{T}} \equiv \mathbf{0}
$$

meaning that the ensemble perturbations and the measurement errors are uncorrelated (equivalent to the common assumption of uncorrelated forecast and measurement errors), then the following is valid

$$
\boldsymbol{H} \boldsymbol{A}^{\prime} \boldsymbol{A}^{\prime \mathrm{T}} \boldsymbol{H}^{\mathrm{T}}+\boldsymbol{r} \boldsymbol{\Upsilon}^{\mathrm{T}}=\left(\boldsymbol{H} \boldsymbol{A}^{\prime}+\boldsymbol{\Upsilon}\right)\left(\boldsymbol{H} \boldsymbol{A}^{\prime}+\boldsymbol{\Upsilon}\right)^{\mathrm{T}}
$$

This is an important point since it means that the inverse can be computed to a cost proportional to $m N$ rather than $m^{2}$ ! This is seen by the following: first compute the singular value decomposition (SVD) of the $m \times N$ matrix

$$
\boldsymbol{H} \boldsymbol{A}^{\prime}+\boldsymbol{r}=\boldsymbol{U} \boldsymbol{\Sigma} \boldsymbol{V}^{\mathrm{T}}
$$

The equation (57) then becomes

$$
\boldsymbol{H} \boldsymbol{A}^{\prime} \boldsymbol{A}^{\prime \mathrm{T}} \boldsymbol{H}^{\mathrm{T}}+\boldsymbol{r} \boldsymbol{r}^{\mathrm{T}}=\boldsymbol{U} \boldsymbol{\Sigma} \boldsymbol{V}^{\mathrm{T}} \boldsymbol{V} \boldsymbol{\Sigma}^{\mathrm{T}} \boldsymbol{U}^{\mathrm{T}}=\boldsymbol{U} \boldsymbol{\Sigma} \boldsymbol{\Sigma}^{\mathrm{T}} \boldsymbol{U}^{\mathrm{T}}
$$

Here the product $\Sigma \Sigma^{\mathrm{T}}$ will be identical to the upper left $N \times N$ quadrant of $\boldsymbol{\Lambda}$ which corresponds to the $N$ non-zero eigenvalues. Further, the $N$ singular vectors contained in $\boldsymbol{U}$ are also identical to the $N$ first eigenvectors in $Z$. Thus, the inverse is again (55). The numerical cost is now proportional to $m N$ which is a huge benefit when $m$ is large. This procedure allows us to efficiently compute the inversion for a global analysis in most practical situations.

Update costs. As soon as the inversion just discussed has been completed, the analysis can be computed from

$$
\boldsymbol{A}^{\mathrm{a}}=\boldsymbol{A}+\boldsymbol{A}^{\prime}\left(\boldsymbol{H} \boldsymbol{A}^{\prime}\right)^{\mathrm{T}} \boldsymbol{U} \boldsymbol{\Lambda}^{-1} \boldsymbol{U}^{\mathrm{T}} \boldsymbol{D}^{\prime}
$$

The matrix $\Lambda^{-1}$ will only have non-zero elements on the diagonal. If we use the pseudo inverse taking into account e.g., $99 \%$ of the variance, only the first few $p \leq N$, terms will be nonzero since the rank of the inverted matrix is $p \leq N$ from (57). This can be exploited using the following scheme:

$$
\begin{array}{llrl}
\boldsymbol{X}_{1}=\boldsymbol{\Lambda}^{-1} \boldsymbol{U}^{\mathrm{T}} & \in \Re^{N \times m} & \mathcal{O}(m p), \\
\boldsymbol{X}_{2}=\boldsymbol{X}_{1} \boldsymbol{D}^{\prime} & \in \Re^{N \times N} & \mathcal{O}(m N p), \\
\boldsymbol{X}_{3}=\boldsymbol{U} \boldsymbol{X}_{2} & \in \Re^{m \times N} & \mathcal{O}(m N p), \\
\boldsymbol{X}_{4}=\left(\boldsymbol{H} \boldsymbol{A}^{\prime}\right)^{\mathrm{T}} \boldsymbol{X}_{3} & \in \Re^{N \times N} & \mathcal{O}(m N N), \\
\boldsymbol{A}^{\mathrm{a}}=\boldsymbol{A}+\boldsymbol{A}^{\prime} \boldsymbol{X}_{4} & \in \Re^{n \times N} & \mathcal{O}(n N N) .
\end{array}
$$

Since $p \leq N$ and $m \ll n$ for all practical applications, the dominant cost is now the last computation which is $n N^{2}$ and which is independent of $m$. All the steps including the singular value decomposition have a cost which is linear in the number of measurements rather than quadratic. A practical approach for performing this last multiplication will be discussed later.

If we use a full rank matrix, $\boldsymbol{H} \boldsymbol{P}_{\mathrm{e}} \boldsymbol{H}^{\mathrm{T}}+\boldsymbol{R}$, where $\boldsymbol{R}$ is not represented using an ensemble of perturbations, the computation of the analysis will be significantly more expensive. First, the full matrix $\boldsymbol{H} \boldsymbol{P}_{\mathrm{e}} \boldsymbol{H}^{\mathrm{T}}=\left(\boldsymbol{H} \boldsymbol{A}^{\prime}\right)\left(\boldsymbol{H} \boldsymbol{A}^{\prime}\right)^{\mathrm{T}}$ must be constructed to a cost of $\mathcal{O}\left(\mathrm{m}^{2} \mathrm{~N}\right)$, followed by the eigenvalue decomposition (54) which requires another $\mathcal{O}\left(\mathrm{m}^{2}\right)$ floating point operations. In this case, the steps (62) and (63) also comes at a cost of $\mathcal{O}\left(\mathrm{m}^{2} \mathrm{~N}\right)$. Thus, the introduction of low rank by representing the measurement error covariance matrix with an ensemble of perturbations, leads to a significant saving by transforming all the $\mathcal{O}\left(m^{2} N\right)$ operations to be linear in $m$.

4.3.3 Remarks on analysis equation Equation (65) expresses the analysis as a first guess plus a linear combination of ensemble perturbations, i.e. $\boldsymbol{A}^{\prime} \boldsymbol{X}_{4}$. From the discussion above we could also write the analysis equation as

$$
\boldsymbol{A}^{\mathrm{a}}=\boldsymbol{A}+\boldsymbol{A}^{\prime}\left(\boldsymbol{H} \boldsymbol{A}^{\prime}\right)^{\mathrm{T}} \boldsymbol{X}_{3}=\boldsymbol{A}+\boldsymbol{P}_{\mathrm{e}} \boldsymbol{H}^{\mathrm{T}}(N-1) \boldsymbol{X}_{3} .
$$

This is the standard notation used in Kalman filters where one measures the error covariance matrix to compute the influence functions, one for each measurement, which are added to the forecast.

Note also that the Equation (65) can be written as

$$
\begin{aligned}
\boldsymbol{A}^{\mathrm{a}} & =\boldsymbol{A}+(\boldsymbol{A}-\overline{\boldsymbol{A}}) \boldsymbol{X}_{4} \\
& =\boldsymbol{A}+\boldsymbol{A}\left(\boldsymbol{I}-\mathbf{1}_{N}\right) \boldsymbol{X}_{4} \\
& =\boldsymbol{A}\left(\boldsymbol{I}+\boldsymbol{X}_{4}\right) \\
& =\boldsymbol{A} \boldsymbol{X}_{5},
\end{aligned}
$$

where we have used that $\mathbf{1}_{N} \boldsymbol{X}_{4} \equiv 0$. Obviously, the first observation to make is that the analyzed ensemble becomes a linear combination of the predicted ensemble. It then becomes of interest to examine $\boldsymbol{X}_{5}$ to study the properties of this particular linear combination. Each column of $\boldsymbol{X}_{5}$ will hold the linear combination defining the corresponding new ensemble member. For this estimate to be unbiased the sum of each column of $\boldsymbol{X}_{5}$ should be equal to one, which is actually a good test for the numerical coding leading to $\boldsymbol{X}_{5}$. Also, one 
can in most applications expect that $\boldsymbol{X}_{5}$ is diagonal dominant since the diagonal holds the coefficient for the first guess ensemble member, while all off-diagonal elements introduces corrections imposed by the measurements. By examining the rows of the matrix $\boldsymbol{X}_{5}$ one can determine if some ensemble members appear to be more important than others. Note that the off-diagonal elements in $\boldsymbol{X}_{5}$ will also have negative values. Thus, the new ensemble members are not weighted averages of the original ensemble, but rather linear combinations.

Computation of the mean of the analyzed ensemble can be written as follows:

$$
\begin{aligned}
\overline{\boldsymbol{\psi}^{\mathrm{a}}} & =\frac{1}{N} \sum_{j=1}^{N} \boldsymbol{\psi}_{j}^{\mathrm{a}}, \\
& =\frac{1}{N} \sum_{j=1}^{N} \sum_{i=1}^{N} \boldsymbol{\psi}_{i} X_{i j}, \\
& =\frac{1}{N} \sum_{i=1}^{N} \boldsymbol{\psi}_{i} \sum_{j=1}^{N} X_{i j}, \\
& =\frac{1}{N} \sum_{i=1}^{N} \boldsymbol{\psi}_{i} y_{i}, \quad \text { where } y_{i}=\sum_{j=1}^{N} X_{i j} .
\end{aligned}
$$

Thus, the sum, $y_{i}$, of the elements in each row in $\boldsymbol{X}_{5}$ defines the coefficients for the linear combination of forecast members defining the mean of the analysis. The $y_{i}$ values therefore also determines which of the ensemble members contributes most strongly to the analysis.

If we compute an SVD decomposition of the forecast ensemble, the equation (65) can be written as

$$
\begin{aligned}
\boldsymbol{A}^{\mathrm{a}} & =\boldsymbol{A} \boldsymbol{X}_{5}, \\
& =\boldsymbol{U} \boldsymbol{\Sigma} \boldsymbol{V}^{\mathrm{T}} \boldsymbol{X}_{5}, \\
& =\boldsymbol{U} \boldsymbol{X}_{6} .
\end{aligned}
$$

Thus, it is possible to visualize the analysis as a linear combination of orthogonal singular vectors. This procedure may be useful since it allows us to reject eventual dependent ensemble members and possibly add new orthogonal members if these are needed. In particular it can be used to examine how linearly independent the ensemble of model states is.

Some interesting conclusions which can be drawn are:

1. The covariances are only used to create the $\boldsymbol{H} \boldsymbol{P} \boldsymbol{H}^{\mathrm{T}}$ matrix, which only includes covariances between the observed variables at the locations of the observations. The actual covariances are never computed when the SVD algorithm in Section 4.3.2 is used although they are used implicitly.

2. The analysis is not really computed as a linear combination of covariance functions. It is in fact computed as a linear combination of the forecasted ensemble members. Each of these members can be considered as drawn from an infinite sample of dynamically consistent model states where the correct multivariate correlations are present in each ensemble member.
3. The covariances are only important for computing the best possible linear combination, i.e., the matrix $\boldsymbol{X}_{5}$. As long as the ensemble based $\boldsymbol{X}_{5}$ is a good approximation, the accuracy of the final analysis will be determined by the relevance and dimension of the space spanned by the ensemble members, relative to the dominant degrees of freedom of the model state.

Based on the points given above it is not wise to filter covariance functions as has been proposed in a number of studies, e.g. by Houtekamer and Mitchell (2001). Clearly, from equation (70), the analysis becomes a linear combination of model states even if equation (66) is used for the actual computation since these equations are identical. However, if equation (66) is used for the computation of the analysis but with filtering applied to the covariance functions, one actually introduces spurious or nondynamical modes in the analysis.

\subsection{Local analysis}

To avoid the problems associated with a large $m$, many operational assimilation schemes have made an assumption that only measurements located within a certain distance from a grid point will impact the analysis in this grid point. This allows for an algorithm where the analysis is computed grid point by grid point. Only a subset of observations, which are located near the current grid point, is used in the analysis for this particular grid point. This algorithm is approximative and it does not solve the original problem posed. Further, it is not clear how serious the approximation is.

Such an approach is not needed for handling a large $m$ in the EnKF if the algorithm just described is used. However, there are other arguments for computing local analyses grid point by grid point. The analysis in the EnKF is computed in a space spanned by the ensemble members. This is a subspace which is rather small compared to the total dimension of the model state. Computing the analysis grid point by grid point implies that a small model state is solved for in a relatively large ensemble space. Further, the analysis will use a different linear combination of ensemble members for each grid point, and this also allows for a larger flexibility in the scheme to reach different model solutions.

For each horizontal grid point, we can now compute the corresponding $\boldsymbol{X}_{5}$ using only the selected measurements contributing to that particular grid point and update the ensemble for that particular grid point. The analysis at grid point $(i, j)$, i.e., $\boldsymbol{A}_{(i, j)}^{\mathrm{a}}$ then becomes

$$
\begin{aligned}
\boldsymbol{A}_{(i, j)}^{\mathrm{a}} & =\boldsymbol{A}_{(i, j)} \boldsymbol{X}_{5,(i, j)} \\
& =\boldsymbol{A}_{(i, j)} \boldsymbol{X}_{5}+\boldsymbol{A}_{(i, j)}\left(\boldsymbol{X}_{5,(i, j)}-\boldsymbol{X}_{5}\right),
\end{aligned}
$$

where $\boldsymbol{X}_{5}$ is the global solution while $\boldsymbol{X}_{5,(i, j)}$ becomes the solution for a local analysis corresponding to grid point $(i, j)$ where only the nearest measurements are used in the analysis. Thus, it is possible to compute the global analysis first, and then add the corrections from the local analysis if these are significant. 
At this time it is not clear how large the ensemble needs to be to explore a large enough part of the state space to provide a more consistent result for the global analysis versus the local analysis scheme. However, we expect this to be application dependent.

\subsection{Nonlinear measurements}

The expression $\boldsymbol{D}^{\prime}=\boldsymbol{D}-\boldsymbol{H} \boldsymbol{A}$ is just the difference between the ensemble of measurements and the ensemble of observed model states. If the observations are nonlinear functions of the model state this matrix formulation using $\boldsymbol{H}$ becomes invalid. The traditional solution is to linearize and iterate. In the EnKF we have the possibility to augment the model state with a diagnostic variable which is the model prediction of the measurement. Thus, if $\boldsymbol{d}=\boldsymbol{h}(\boldsymbol{\psi}, \ldots)+\boldsymbol{\epsilon}$ then a new model state can be defined for each ensemble member as

$$
\widehat{\boldsymbol{\psi}}^{\mathrm{T}}=\left(\boldsymbol{\psi}^{\mathrm{T}}, \boldsymbol{h}^{\mathrm{T}}(\boldsymbol{\psi}, \ldots)\right)
$$

By defining the new ensemble matrix as

$$
\widehat{\boldsymbol{A}}=\left(\widehat{\boldsymbol{\psi}}_{1}, \widehat{\boldsymbol{\psi}}_{2}, \ldots, \widehat{\boldsymbol{\psi}}_{N}\right) \in \Re^{\hat{n} \times N},
$$

with $\hat{n}$ being the $n$ plus the number of measurement equivalents added to the original model state, the analysis can be written

$$
\boldsymbol{A}^{\mathrm{a}}=\boldsymbol{A}+\boldsymbol{A}^{\prime} \widehat{\boldsymbol{A}}^{\prime \mathrm{T}} \widehat{\boldsymbol{H}}^{\mathrm{T}}\left(\widehat{\boldsymbol{H}} \widehat{\boldsymbol{A}}^{\prime} \widehat{\boldsymbol{A}}^{\prime \mathrm{T}} \widehat{\boldsymbol{H}}^{\mathrm{T}}+\boldsymbol{r} \boldsymbol{r}^{\mathrm{T}}\right)^{-1} \boldsymbol{D}^{\prime}
$$

where the now linear innovations (with $\widehat{\boldsymbol{H}}$ being a direct and linear measurement functional) becomes

$$
D^{\prime}=D-\widehat{H} \widehat{A} .
$$

From this expression, where the ensemble members have been augmented with the observation equivalent, we can compute the following: the innovation $\boldsymbol{D}^{\prime}$ using a linear (direct) measurement functional; the model predicted error covariance of the observation's equivalents $\boldsymbol{H} \widehat{\boldsymbol{A}}^{\prime} \widehat{\boldsymbol{A}}^{\prime \mathrm{T}} \boldsymbol{H}^{\mathrm{T}}$; and the covariance between the observations and all prognostic model variables from $\boldsymbol{A}^{\prime} \widehat{\boldsymbol{A}}^{\mathrm{T}} \widehat{\boldsymbol{H}}^{\mathrm{T}}$.

The analysis is a linear combination of model predicted error covariances between the observation equivalents $\boldsymbol{h}(\boldsymbol{\psi}, \ldots$ and all other model variables. Thus, we have a fully multivariate analysis scheme.

\subsection{Assimilation of "non-synoptic” measurements}

In some cases measurements occur with high frequency in time. An example is along track satellite data. It is not practical to perform an analysis every time there is a measurement. Further, the normal approach of assimilating, at one time instant, all data collected within a time interval, is not optimal. Based on the theory from Evensen and van Leeuwen (2000), it is possible to assimilate the non-synoptic measurements at one time instant by exploiting the time correlations in the ensemble. Thus, a measurement collected at a previous time allows for the computation of the $\boldsymbol{H} \boldsymbol{A}$ at that time and thereby also the innovations. By treating these as augmented model variables the equation (82) can again be used but with the $\boldsymbol{h}(\boldsymbol{\psi}, \ldots)$ now denoting the measurements collected at earlier times.

\section{Numerical implementation of the EnKF}

The algorithm as explained in the previous sections provides an optimal approach for computing the EnKF analysis. The following provides a basis explaining the implementation of the EnKF analysis scheme. It assumes access to the BLAS, LAPACK and EISPACK libraries, where highly optimized numerical subroutines are available for most computer systems and which can be obtained for free through www. net lib. no.

\subsection{Storing the ensemble on disk}

For most practical applications one will not want to keep the whole ensemble in memory during the ensemble integrations. Rather, an approach where ensemble members are kept in a file residing on disk is convenient. This allows for the system to read a particular member from file, integrate it forward in time, and then store it on disk again following the integration. An approach where each member is stored in a record in a direct Fortran file is most convenient. This allows us to read and write specific records containing individual ensemble members.

\subsection{Analysis implementation}

The algorithm for the analysis exploits that we can compute once and store all innovations, measurement perturbations and the measurements of the ensemble. Thus, we start with the following:

1. Read the whole ensemble forecast into $\boldsymbol{A}$.

2. Compute the matrix $\boldsymbol{H} \boldsymbol{A}$.

3. Compute the measurement perturbations $\boldsymbol{\Upsilon}$.

4. Compute the innovations $\boldsymbol{D}^{\prime}$.

5. Compute $\overline{\boldsymbol{H A}}$ and subtract it from $\boldsymbol{H} \boldsymbol{A}$ to get $\boldsymbol{H} \boldsymbol{A}^{\prime}$ (requires $\boldsymbol{H}$ to be linear).

The following subroutine can then be used without any modification to compute the analysis for all kinds of model states.

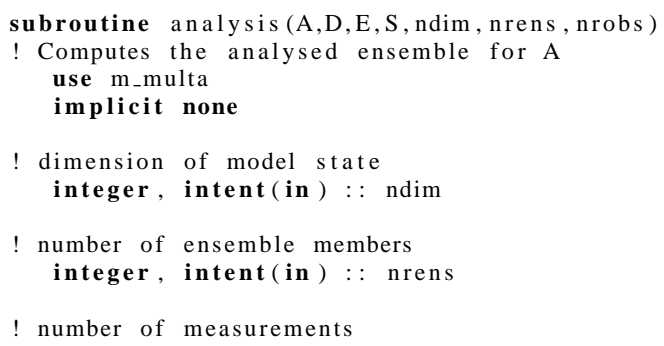




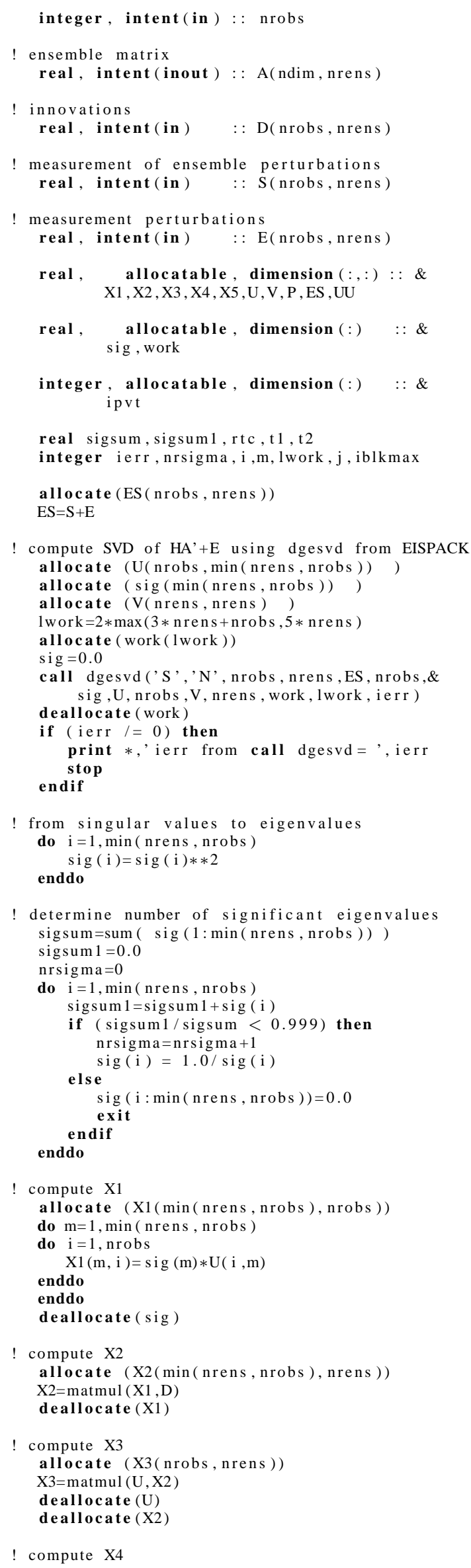

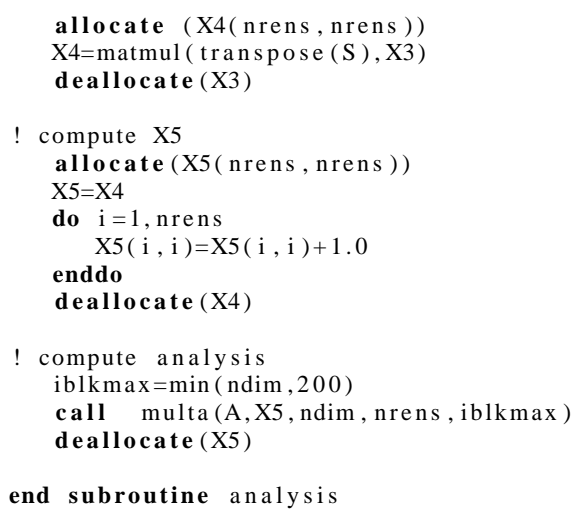

\subsection{Final update}

The most demanding step in the EnKF analysis is the final step when evaluating the analysis ensemble from equation (70). The largest matrix to be held in memory is the ensemble matrix $\boldsymbol{A} \in \Re^{n \times N}$. Further, the number of floating point operations (a multiply and add) is $n N^{2}$ which is likely to be several orders of magnitude more than for the previous steps in the algorithm. Thus, this step will be treated in some detail.

It is possible to develop a multiplication algorithm which only requires one copy of the ensemble to be kept in memory. This matrix multiplication can easily be done while overwriting row by row of $\boldsymbol{A}$ using the following call to the subroutine multa using

$$
\boldsymbol{X}_{5}=\boldsymbol{I}+\boldsymbol{X}_{4} \in \Re^{N \times N} .
$$

The following subroutine has been found to perform this multiplication very efficiently. It uses optimized BLAS routines and includes a block representation which can be used to optimize cache performance.

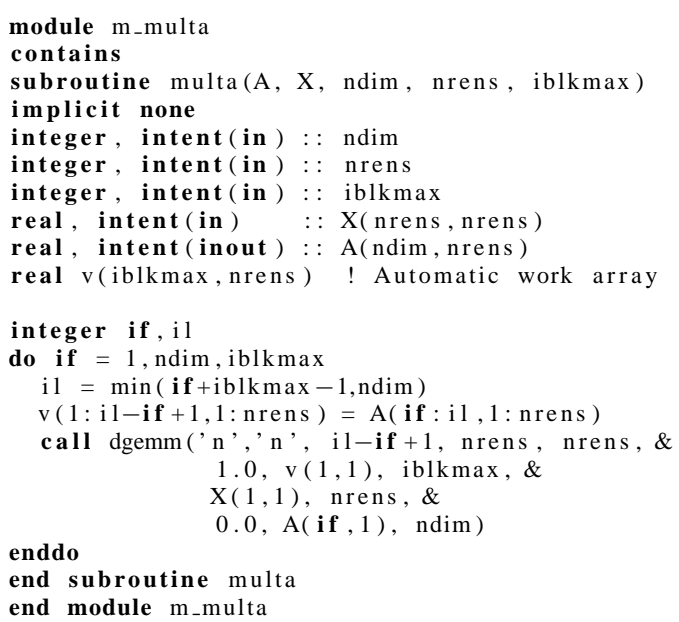

5.3.1 Remark 1 Note that this routine does not care about the order of which the elements in A are stored for each ensemble member. Thus, in the call to multa, A can be a multi dimensional matrix e.g., $A(n x, n y, n z, n r e n s)$ holding an ensemble of a uni-variate three dimensional model state. A multi-variate model state can be stored in a structure or type declaration, and still be input to multa. 
5.3.2 Remark 2: In principle the multiplication has a serious drawback caused by the stride ndim copies. Here the routine relies on BLAS for the inner matrix multiplication, since the BLAS routines have already been designed to optimize cache performance. The variable $i b l \mathrm{kmax}$ is only used for storage considerations and a typical value of 200 seems to work fine. This routine also opens for a possible block representation of the model state.

\subsection{A block algorithm for large ensemble matrices}

It is still possible to use the EnKF even if the whole ensemble does not fit in memory. In this case a block algorithm can be used for the final update, using additional reads and writes to file. The algorithm goes as follows:

1. Read each individual ensemble member into a vector one at the time while computing and storing the columns of $\boldsymbol{H} \boldsymbol{A}$.

2. Compute the measurement perturbations $\boldsymbol{\Upsilon}$.

3. Compute the innovations $\boldsymbol{D}^{\prime}$.

4. Compute $\overline{\boldsymbol{H} \boldsymbol{A}}$ and subtract it from $\boldsymbol{H} \boldsymbol{A}$ to get $\boldsymbol{H} \boldsymbol{A}^{\prime}$ (requires $\boldsymbol{H}$ to be linear).

So far we have only kept one full model state in memory at the time. It remains to solve the Equation (70). Using the block algorithm just discussed above it is possible to perform this computation without keeping all of the ensemble in memory at once. A proposed strategy would be to store the ensemble in several files, say one file for the temperature, one for the salinity, etc. Then the analysis can be done easily by first one read of the part of the ensemble that is required for the computation of $\boldsymbol{H} \boldsymbol{A}$, followed by one additional read and write of the whole ensemble, i.e., to update the full ensemble based on the information in $\boldsymbol{H} \boldsymbol{A}$.

\section{A Consistency checks on error statistics}

The EnKF provides error statistics for the results. To validate the predicted error statistics it is possible to compare statistics computed from the innovation sequence with the predicted error statistics.

If the model forecast is written as

$$
\psi^{\mathrm{f}}=\boldsymbol{\psi}^{\mathrm{t}}+\boldsymbol{q}
$$

i.e., it is given as the truth plus an error, and the measurements are written as

$$
\boldsymbol{d}=\boldsymbol{H} \boldsymbol{\psi}^{\mathrm{t}}+\boldsymbol{\epsilon}
$$

the innovation becomes

$$
\boldsymbol{d}-\boldsymbol{H} \psi^{\mathrm{f}}=\boldsymbol{\epsilon}-\boldsymbol{H} \boldsymbol{q}
$$

By squaring this equation and taking the expectation we get the expression

$$
\overline{\left(\boldsymbol{d}-\boldsymbol{H} \boldsymbol{\psi}^{\mathrm{f}}\right)\left(\boldsymbol{d}-\boldsymbol{H} \boldsymbol{\psi}^{\mathrm{f}}\right)^{\mathrm{T}}}=\boldsymbol{R}+\boldsymbol{H} \boldsymbol{P}^{\mathrm{f}} \boldsymbol{H}^{\mathrm{T}}
$$

where correlations between the forecast error and the measurement error have been neglected.

Thus, it is possible to compute the variance of the innovation sequence in time, subtract the measurement variance and compare this with the predicted error variance from the ensemble. This provides a solid consistency test on the prescribed error statistics used in the EnKF.

\section{B Ensemble Optimal Interpolation (EnOI)}

Traditional optimal interpolation (OI) schemes have estimated or prescribed covariances using an ensemble of model states which has been sampled during a long time integration. Normally the estimated covariances are fitted to simple functional forms which are used uniformly throughout the model grid.

Based on the discussion in this paper it is natural to device an OI scheme where the analysis is computed in the space spanned by a stationary ensemble of model states sampled, e.g., during a long time integration. This approach is denoted Ensemble OI (EnOI).

The EnOI analysis is computed by solving an equation similar to (89) but written as

$$
\boldsymbol{\psi}^{\mathrm{a}}=\boldsymbol{\psi}+\alpha \boldsymbol{A}^{\prime} \boldsymbol{A}^{\prime \mathrm{T}} \boldsymbol{H}^{\mathrm{T}}\left(\alpha \boldsymbol{H} \boldsymbol{A}^{\prime} \boldsymbol{A}^{\prime \mathrm{T}} \boldsymbol{H}^{\mathrm{T}}+\boldsymbol{r} \boldsymbol{\Upsilon}^{\mathrm{T}}\right)^{-1}(\boldsymbol{d}-\boldsymbol{H} \boldsymbol{\psi}) .
$$

The analysis is now computed for only one single model state, and a parameter $\alpha \in(0,1]$ is introduced to allow for different weights on the ensemble versus measurements. Naturally, an ensemble consisting of model states sampled over a long time period will have a climatological variance which is too large to represent the actual error in the model forecast, and $\alpha$ is used to reduce the variance to a realistic level.

The practical implementation introduces $\alpha$ in (58), which is now written as

$$
\sqrt{\alpha} \boldsymbol{H} \boldsymbol{A}^{\prime}+\boldsymbol{\Upsilon}=\boldsymbol{U} \boldsymbol{\Sigma} \boldsymbol{V}^{\mathrm{T}}
$$

and the coefficient matrix $\boldsymbol{X}_{4}$ in (64) is further scaled with $\alpha$ before $\boldsymbol{X}_{5}$ is computed.

The EnOI method allows for the computation of a multivariate analysis in dynamical balance, just like the EnKF. However, a larger ensemble may be useful to ensure that it spans a large enough space to properly represent the correct analysis.

The EnOI is an attractive approach to save computer time. Once the stationary ensemble is created, only one single model integration is required in addition to the analysis step where the final update cost is reduced to $\mathcal{O}(n N)$ because only one model state is updated. The method is numerically extremely efficient but it will always provide a suboptimal solution compared to the EnKF. In addition it does not provide consistent error estimates for the solution.

\section{Assimilation of in situ measurements}

The direct assimilation of in situ observations such as temperature and salinity profiles poses a problem in all ocean mod- 
els unless both temperature and salinity is known simultaneously. It is not known, a priori, how to update the water-mass properties in a consistent manner, see e.g., Troccoli et al. (2002) and Thacker and Esenkov (2002).

A new solution is proposed here. It is based on the definition of a measurement functional which interpolates the model temperature and/or salinity to the measurement location in depth. The spline interpolation algorithm discussed below is probably close to the best alternative of a measurement functional for layered models.

The results from the discussion on nonlinear measurement functionals allow us to augment the model state with observation equivalents for each independent in situ measurement. Further, the "global" analysis algorithm allows us to handle a large number of profiles of measurements simultaneously still to a low numerical cost. This approach ensures that the model update in the vertical and horizontal is performed consistently with the error statistics predicted by the ensemble.

In order to obtain a variable's value at a specific depth an interpolation algorithm is needed. We used a second order spline to interpolate in the vertical. It is important to note that when interpolating values between different layers the interpolating spline should not pass exactly through the mean of the variable at the center of each layer. Instead a criterion is used where the mean value computed by integrating the spline function across the layer is equal to the mean of the variable in that layer. The details of the algorithm follows.

\section{C.1 Upper layer}

Layer one is divided into upper and lower parts where the spline polynomial, used to represent the variable to be interpolated is defined as

$$
f_{1}(x)=\left\{\begin{array}{lll}
c_{0} & \text { for } & x \in\left[0, \frac{1}{2} h_{1}\right) \\
a_{1} x^{2}+b_{1} x+c_{1} & \text { for } & x \in\left[\frac{1}{2} h_{1}, h_{1}\right] .
\end{array}\right.
$$

Here $h_{i}$ is the location of the lower interface of layer $i$. Conditions are specified at $x=\frac{1}{2} h_{1}$ for continuity of the function and the derivative, i.e.,

$$
f_{1}\left(\frac{1}{2} h_{1}\right)=c_{0},
$$

and

$$
\left.\frac{\partial f_{1}(x)}{\partial x}\right|_{\frac{1}{2} h_{1}}=0
$$

and in addition the integral over layer 1 should satisfy

$$
\frac{1}{h_{1}} \int_{0}^{h_{1}} f_{1}(x)=c_{0} \frac{1}{2}+a_{1} \frac{7}{24} h_{1}^{2}+b_{1} \frac{3}{8} h_{1}+c_{1} \frac{1}{2}=\bar{u}_{1},
$$

with $\bar{u}_{1}$ being the model predicted layer variable in layer one.

\section{C.2 Interior layers}

Within each interior layer, $i$, a function of the form

$$
f_{i}(x)=a_{i} x^{2}+b_{i} x+c_{i},
$$

is used to represent the model variables. For each interior layer there are three conditions which determine the three unknowns in each layer, i.e., continuity at layer interfaces

$$
f_{i}\left(h_{i-1}\right)=f_{i-1}\left(h_{i-1}\right),
$$

continuity of derivatives at layer interfaces

$$
\left.\frac{\partial f_{i}(x)}{\partial x}\right|_{h_{i-1}}=\left.\frac{\partial f_{i-1}(x)}{\partial x}\right|_{h_{i-1}},
$$

and a condition for the mean of the variable becomes after some manipulations

$$
\begin{gathered}
\frac{1}{h_{i}-h_{i-1}} \int_{h_{i-1}}^{h_{i}} f_{i}(x) d x=a_{i} \frac{1}{3}\left(h_{i-1}^{2}+h_{i-1} h_{i}+h_{i}^{2}\right) \\
+b_{i} \frac{1}{2}\left(h_{i}+h_{i-1}\right)+c_{i}=\bar{u}_{i}
\end{gathered}
$$

\section{C.3 Closing the system}

A final condition is obtained by setting the variable at the sea floor equal to the mean of the variable in the bottom layer,

$$
f_{k}\left(h_{k}\right)=\bar{u}_{k}
$$

Thus, the system is closed.

\section{Ensemble Kalman Smoother (EnKS)}

In light of the discussion in this paper it is also possible to device an efficient implementation of the EnKS. The EnKS, as described in Evensen and van Leeuwen (2000), updates the ensemble at prior times every time new measurements are available. The update exploits the space-time correlations between the model forecast at measurement locations and the model state at a prior time. It allows for a sequential processing of the measurements in time. Thus, every time a new set of measurements becomes available the ensemble at the current and all prior times can be updated.

Similar to the analysis equation (89) the analysis for a prior time $t^{\prime}$ which results from the introduction of a new measurement vector at time $t>t^{\prime}$ can be written as

$$
\begin{aligned}
\boldsymbol{A}^{\mathrm{a}}\left(t^{\prime}\right) & =\boldsymbol{A}\left(t^{\prime}\right)+\boldsymbol{A}^{\prime}\left(t^{\prime}\right) \boldsymbol{A}^{\prime \mathrm{T}}(t) \boldsymbol{H}^{\mathrm{T}} \\
& \left(\boldsymbol{H} \boldsymbol{A}^{\prime}(t) \boldsymbol{A}^{\prime \mathrm{T}}(t) \boldsymbol{H}^{\mathrm{T}}+\boldsymbol{r} \boldsymbol{r}^{\mathrm{T}}\right)^{-1} \boldsymbol{D}^{\prime}(t),
\end{aligned}
$$

This equation is updated repetitively every time a new set of measurements are introduced at future times $t$.

The EnKS analysis can best be computed using the formulation discussed in the previous sections, and in particular using the definition of $\boldsymbol{X}_{5}$ in Equation (84). It is easily seen 
that the linear combination $\boldsymbol{X}_{5}(t)$ corresponding to the measurements at time $t$, is also used on the analysis ensemble at the prior times $t^{\prime}$ to update the smoother estimate at time $t^{\prime}$.

Thus, the smoother estimate at a time $t^{\prime}$ where $t_{i-1} \leq$ $t^{\prime}<t_{i} \leq t_{k}$, using future data from the data times $\left(t_{i}, t_{i+1}, \ldots, t_{k}\right.$ is just

$$
\boldsymbol{A}_{\mathrm{EnKS}}^{\mathrm{a}}\left(t^{\prime}\right)=\boldsymbol{A}_{\mathrm{EnKF}}\left(t^{\prime}\right) \prod_{j=i}^{k} \boldsymbol{X}_{5}\left(t_{j}\right) .
$$

As long as the previous ensemble files have been stored, it is straight forward to update them with new information every time a new set of measurements is available and the matrix $\boldsymbol{X}_{5}$ corresponding to these measurements have been computed. This discussion has assumed that a global analysis is used. The local analysis becomes a little less practical since there is an $\boldsymbol{X}_{5}$ matrix for each grid point.

The product in Equation (101) has an important property. The multiplication of the ensemble with $\boldsymbol{X}_{5}$ will always result in a new ensemble with a different mean and a smaller variance. Thus, each consecutive update through the repetitive multiplication in (101) will lead to slight reduction of variance and slight change of mean. Eventually, there will be a convergence with only negligible updates of the ensemble when measurements are taken further into the future than the actual decorrelation time.

\section{E Generating pseudo random fields}

Here a procedure is given which can be used to compute smooth pseudo random fields with mean equal to zero, variance equal to one, and a specified covariance which determines the smoothness of the fields. The algorithm follows the presentation in the appendix of Evensen (1994a), and additional details and explanations are given by Natvik (2001).

Let $q=q(x, y)$ be a continuous field, which may be described by its Fourier transform

$$
q(x, y)=\int_{-\infty}^{\infty} \int_{-\infty}^{\infty} \widehat{q}(\mathbf{k}) e^{i \mathbf{k} \cdot \mathbf{x}} d \mathbf{k} .
$$

Now, we are using an $N \times M$ grid. Further, we define $\mathbf{k}=$ $\left(\kappa_{l}, \gamma_{p}\right)$, where $l$ and $p$ are counters and $\kappa_{l}$ and $\gamma_{p}$ are wave numbers in the $N$ and $M$ directions, respectively. We now get a discrete version of equation (102),

$$
q\left(x_{n}, y_{m}\right)=\sum_{l, p} \widehat{q}\left(\kappa_{l}, \gamma_{p}\right) e^{i\left(\kappa_{l} x_{n}+\gamma_{p} y_{m}\right)} \Delta \mathbf{k}
$$

where $x_{n}=n \Delta x$ and $y_{m}=m \Delta y$. For the wave numbers, we have

$$
\begin{gathered}
\kappa_{l}=\frac{2 \pi l}{x_{N}}=\frac{2 \pi l}{N \Delta x}, \\
\gamma_{P}=\frac{2 \pi p}{y_{M}}=\frac{2 \pi p}{M \Delta y} \\
\Delta \mathbf{k}=\Delta \kappa \Delta \gamma=\frac{(2 \pi)^{2}}{N M \Delta x \Delta y} .
\end{gathered}
$$

We define (assume) the following form of $\widehat{q}(\mathbf{k})$ :

$$
\widehat{q}\left(\kappa_{l}, \gamma_{p}\right)=\frac{c}{\sqrt{\Delta \mathbf{k}}} e^{-\left(\kappa_{l}^{2}+\gamma_{p}^{2}\right) / \sigma^{2}} e^{2 \pi i \phi_{l, p}},
$$

$\left.t_{k}\right)$ where $\phi_{l, p} \in[0,1]$ is a random number which introduces a random phase shift. (The exponential function may be written as a sum of sine and cosine terms). Note that increasing wave numbers $\kappa_{l}$ and $\gamma_{p}$ will give an exponentially decreasing contribution to the expression above. Now, equation (107) may be inserted into equation (103), and we get

$$
\begin{aligned}
& q\left(x_{n}, y_{m}\right)= \\
& \sum_{l, p} \frac{c}{\sqrt{\Delta \mathbf{k}}} e^{-\left(\kappa_{l}^{2}+\gamma_{p}^{2}\right) / \sigma^{2}} e^{2 \pi i \phi_{l, p}} e^{i\left(\kappa_{l} x_{n}+\gamma_{p} y_{m}\right)} \Delta \mathbf{k} .
\end{aligned}
$$

We want equation (108) to produce real fields only. Thus, when the summation over $l, p$ is performed, all the imaginary contributions must add up to zero. This is satisfied whenever

$$
\widehat{q}\left(\kappa_{l}, \gamma_{p}\right)=\widehat{q}^{*}\left(\kappa_{-l}, \gamma_{-p}\right)
$$

where the star denote complex conjugate, and

$$
\operatorname{Im} \widehat{q}\left(\kappa_{0}, \gamma_{0}\right)=0 .
$$

The formula (108) can be used to generate an ensemble of pseudo random fields with a specific covariance determined by the parameters $c$ and $\sigma$. An expression for the covariance is given by

$$
\begin{aligned}
& \overline{q\left(x_{1}, y_{1}\right) q\left(x_{2}, y_{2}\right)}= \\
& \sum_{l, p, r, s} \widehat{q}\left(\kappa_{l}, \gamma_{p}\right) \widehat{q}\left(\kappa_{r}, \gamma_{s}\right) e^{i\left(\kappa_{l} x_{1}+\gamma_{p} y_{1}+\kappa_{r} x_{2}+\gamma_{s} y_{2}\right)}(\Delta \mathbf{k})^{2}
\end{aligned}
$$

By using equation (109), and by noting that the summation goes over both positive and negative $r$ and $s$, we may insert the complex conjugate instead, i. e.

$$
\begin{aligned}
& \overline{q\left(x_{1}, y_{1}\right) q\left(x_{2}, y_{2}\right)} \\
& =\sum_{l, p, r, s} \widehat{q}\left(\kappa_{l}, \gamma_{p}\right) \widehat{q}^{*}\left(\kappa_{r}, \gamma_{s}\right) e^{i\left(\kappa_{l} x_{1}-\kappa_{r} x_{2}+\gamma_{p} y_{1}-\gamma_{s} y_{2}\right)}(\Delta \mathbf{k})^{2} \\
& =\sum_{l, p, r, s} \Delta \mathbf{k} c^{2} e^{-\left(\kappa_{l}^{2}+\gamma_{p}^{2}+\kappa_{r}^{2}+\gamma_{s}^{2}\right) / \sigma^{2}} e^{2 \pi i\left(\phi_{l, p}-\phi_{r, s}\right)} \\
& e^{i\left(\kappa_{l} x_{1}-\kappa_{r} x_{2}+\gamma_{p} y_{1}-\gamma_{s} y_{2}\right)} .
\end{aligned}
$$

We assume that the fields are $\delta$ - correlated in wave space. That is, we assume that there is a distance dependence only (isotropy), and we may set $l=r$ and $p=s$, and the above expression becomes

$$
\begin{aligned}
& \overline{q\left(x_{1}, y_{1}\right) q\left(x_{2}, y_{2}\right)} \\
& =\Delta \mathbf{k} c^{2} \sum_{l, p} e^{-2\left(\kappa_{l}^{2}+\gamma_{p}^{2}\right) / \sigma^{2}} e^{i\left(\kappa_{l}\left(x_{1}-x_{2}\right)+\gamma_{p}\left(y_{1}-y_{2}\right)\right)} .
\end{aligned}
$$

From this equation, the variance at $(x, y)$ is

$$
\overline{q(x, y) q(x, y)}=\Delta \mathbf{k} c^{2} \sum_{l, p} e^{-2\left(\kappa_{l}^{2}+\gamma_{p}^{2}\right) / \sigma^{2}} .
$$


Now, we require the variance to be equal to 1 . Further, we define a decorrelation length $r_{h}$, and we require the covariance corresponding to $r_{h}$ to be equal to $e^{-1}$. For the variance, we get the equation

$$
1=\Delta \mathbf{k} c^{2} \sum_{l, p} e^{-2\left(\kappa_{l}^{2}+\gamma_{p}^{2}\right) / \sigma^{2}},
$$

which means that

$$
c^{2}=\frac{1}{\Delta \mathbf{k} \sum_{l, p} e^{-2\left(\kappa_{l}^{2}+\gamma_{p}^{2}\right) / \sigma^{2}}} .
$$

If we let $x_{1}-x_{2}=r_{h}$ and $y_{1}-y_{2}=0$, we must have a covariance equal to $e^{-1}$ between these points, i. e.,

$$
\begin{aligned}
e^{-1} & =\Delta \mathbf{k} c^{2} \sum_{l, p} e^{-2\left(\kappa_{l}^{2}+\gamma_{p}^{2}\right) / \sigma^{2}} e^{i \kappa_{l} r_{h}} \\
& =\Delta \mathbf{k} c^{2} \sum_{l, p} e^{-2\left(\kappa_{l}^{2}+\gamma_{p}^{2}\right) / \sigma^{2}} \cos \left(\kappa_{l} r_{h}\right)
\end{aligned}
$$

By inserting for $c^{2}$ from equation (116), we get

$$
e^{-1}=\frac{\sum_{l, p} e^{-2\left(\kappa_{l}^{2}+\gamma_{p}^{2}\right) / \sigma^{2}} \cos \left(\kappa_{l} r_{h}\right)}{\sum_{l, p} e^{-2\left(\kappa_{l}^{2}+\gamma_{p}^{2}\right) / \sigma^{2}}}
$$

This is a nonlinear scalar equation for $\sigma$, which may be solved using some numerical routine. One can thereafter find a value for $c$ from equation (116).

Once the values for $c$ and $\sigma$ have been determined, equation (108) may be used to create an ensemble of pseudo random fields with variance 1 and covariance determined by the decorrelation length $r_{h}$. An efficient approach for finding the inverse transform in (108) is to use two-dimensional fast Fourier transform routines (FFT). The inverse FFT is calculated on a grid which is a few characteristic lengths larger than the computational domain to ensure non-periodic fields in the subdomain corresponding to the computational domain (Evensen, 1994a).

To summarize, we are now able to generate (sample) twodimensional pseudo random fields with variance equal to one and a prescribed covariance (isotropic as a function of grid indices). The simple formulas used in Section 4.2 can be used to introduce correlations between the fields.

Acknowledgements I would like to express my thanks and gratitude to co-workers at the Nansen Center and elsewhere for providing valuable inputs and stimulating discussions during the work with this paper. In particular I would like to thank L. Bertino, K. A. Lisæter, Y. Morel, L. J. Natvik, D. Obaton, and H. Sagen, who have helped implementing and testing the new algorithms, checked derivations and contributed to making this paper readable and hopefully useful for the community developing ensemble methods for data assimilation. This work was supported by the EC FP-5 TOPAZ (EVK3-CT2000-00032) and ENACT (EVK2-CT-2001-00117) projects, and has received support from The Research Council of Norway (Programme for Supercomputing) through a grant of computing time.

\section{References}

Allen JI, Eknes M, Evensen G (2002) An Ensemble Kalman Filter with a complex marine ecosystem model: Hindcasting phytoplankton in the Cretan Sea. Annales Geophysicae 20: $1-13$

Anderson JL (2001) An ensemble adjustment Kalman filter for data assimilation. Mon Weather Rev 129: 2884-2903

Anderson JL, Anderson SL (1999) A Monte Carlo implementation of the nonlinear filtering problem to produce ensemble assimilations and forecasts. Mon Weather Rev 127: 2741-2758

Bennett AF (1992) Inverse Methods in Physical Oceanography. Cambridge University Press

Bennett AF, Chua BS (1994) Open-ocean modeling as an inverse problem: The primitive equations. Mon Weather Rev 122: 1326-1336

Bennett AF, Chua BS, Leslie LM (1996) Generalized inversion of a global numerical weather prediction model. Meteorol Atmos Phys 60: 165-178

Bennett AF, Leslie LM, Hagelberg CR, Powers PE (1993) Tropical cyclone prediction using a barotropic model initialized by a generalized inverse method. Mon Weather Rev 121: 1714-1729

Bertino L, Evensen G, Wackernagel H (2002) Combining geostatistics and Kalman filtering for data assimilation in an estuarine system. Inverse Methods 18: 1-23

Bishop CH, Etherton BJ, Majumdar SJ (2001) Adaptive sampling with the ensemble transform Kalman filter. Part I: Theoretical aspects. Mon Weather Rev 129: 420-436

Bleck R, Rooth C, Hu D, Smith LT (1992) Salinity-driven thermohaline transients in a wind- and thermohalineforced isopycnic coordinate model of the North Atlantic. J Phys Oceanogr 22: 1486-1515

Brasseur P, Ballabrera J, Verron J (1999) Assimilation of altimetric data in the mid-latitude oceans using the SEEK filter with an eddy-resolving primitive equation model. J Marine Sys 22: 269-294

Brusdal K, Brankart J, Halberstadt G, Evensen G, Brasseur P, van Leeuwen PJ, Dombrowsky E, Verron J (2002) An evaluation of ensemble based assimilation methods with a layered OGCM. J Marine Sys In print

Burgers G, van Leeuwen PJ, Evensen G (1998) Analysis scheme in the ensemble Kalman Filter. Mon Weather Rev 126: 1719-1724

Carmillet V, Brankart JM, Brasseur P, Drange H, Evensen G (2001) A singular evolutive extended Kalman filter to assimilate ocean color data in a coupled physicalbiochemical model of the North Atlantic. Ocean Modelling 3: 167-192

Courtier P (1997) Dual formulation of four-dimensional variational assimilation. Q J R Meteorol Soc 123: 2449-2461

Courtier P, Talagrand O (1987) Variational assimilation of meteorological observations with the adjoint vorticity equation II: Numerical results. Q J R Meteorol Soc 113: 1329-1347 
Courtier P, Thepaut, Hollingsworth A (1994) A strategy for operational implementation of 4D-Var, using an incremental approach. Q J R Meteorol Soc 120: 1367-1387

Echevin V, Mey PD, Evensen G (2000) Horizontal and vertical structure of the representer functions for sea surface measurements in a coastal circulation model. J Phys Oceanogr 30: 2627-2635

Eknes M, Evensen G (2002) An Ensemble Kalman Filter with a 1-D Marine Ecosystem Model. J Marine Sys 36: 75-100

Evensen G (1992) Using the extended Kalman filter with a Multilayer Quasi-Geostrophic Ocean Model. J Geophys Res 97(C11): 17,905-17,924

Evensen G (1994a) Inverse Methods and data assimilation in nonlinear ocean models. Physica D 77: 108-129

Evensen G (1994b) Sequential data assimilation with a nonlinear quasi-geostrophic model using Monte Carlo methods to forecast error statistics. J Geophys Res 99(C5): $10,143-10,162$

Evensen G (1997) Advanced data assimilation for strongly nonlinear dynamics. Mon Weather Rev 125: 1342-1354

Evensen G (2002) Sequential Data Assimilation for Nonlinear Dynamics: The Ensemble Kalman Filter. In: Pinardi N, Woods JD (eds.) Ocean Forecasting: Conceptual basis and applications, Springer-Verlag Berlin Heidelberg, pp. 97116

Evensen G, van Leeuwen PJ (1996) Assimilation of Geosat Altimeter Data for the Agulhas Current using the Ensemble Kalman Filter with a Quasi-Geostrophic Model. Mon Weather Rev 124: 85-96

Evensen G, van Leeuwen PJ (2000) An Ensemble Kalman Smoother for nonlinear dynamics. Mon Weather Rev 128: 1852-1867

Gradshteyn IS, Ryzhik IM (1979) Table of Integrals, Series, and Products: Corrected and enlarged edition. Academic Press, Inc.

Gronnevik R, Evensen G (2001) Application of ensemble based techniques in fish-stock assessment. Sarsia 86: 517526

Hamill TM, Mullen SL, Snyder C, Toth Z, Baumhefner DP (2000) Ensemble forecasting in the short to medium range: Report from a workshop. Bull Amer Meteor Soc 81(11): 2653-2664

Hamill TM, Snyder C (2000) A hybrid Ensemble Kalman Filter-3D variational analysis scheme. Mon Weather Rev 128: 2905-2919

Hamill TM, Whitaker JS, Snyder C (2001) Distancedependent filtering of background error covariance estimates in an Ensemble Kalman Filter. Mon Weather Rev 129: 2776-2790

Hansen JA, Smith LA (2001) Probabilistic noise reduction. Tellus, Ser A 53: 585-598

Haugen VE, Evensen G (2002) Assimilation of SLA and SST data into an OGCM for the Indian ocean. Ocean Dynamics 52: $133-151$

Haugen VE, Evensen G, Johannessen OM (2002) Indian Ocean circulation: An integrated model and remote sensing study. J Geophys Res 107(C5): 11-1-11-23
Heemink AW, Verlaan M, Segers AJ (2001) Variance reduced ensemble Kalman Filtering. Mon Weather Rev 129: 1718 1728

Houtekamer PL, Mitchell HL (1998) Data Assimilation using an Ensemble Kalman Filter Technique. Mon Weather Rev 126: 796-811

Houtekamer PL, Mitchell HL (1999) Reply. Mon Weather Rev 127: 1378-1379

Houtekamer PL, Mitchell HL (2001) A sequential ensemble Kalman filter for Atmospheric data assimilation. Mon Weather Rev 129: 123-137

Jazwinski AH (1970) Stochastic Processes and Filtering Theory. Academic Press, San Diego, Calif.

Keppenne CL (2000) Data assimilation into a Primitiveequation model with a parallel Ensemble Kalman Filter. Mon Weather Rev 128: 1971-1981

Keppenne CL, Rienecker MM (2002) Initial testing of a massively parallel Ensemble Kalman Filter with the Poseidon isopycnal ocean general circulation model. Mon Weather Rev 130: 2951-2965

Lermusiaux PFJ (2001) Evolving the subspace of the threedimensional ocean variability: Massachusetts Bay. J Marine Sys 29: 385-422

Lermusiaux PFJ, Robinson AR (1999a) Data assimilation via error subspace statistical estimation. Part I: Theory and schemes. Mon Weather Rev 127: 1385-1407

Lermusiaux PFJ, Robinson AR (1999b) Data assimilation via error subspace statistical estimation. Part II: Middle Atlantic Bight shelfbreak front simulations and ESSE validation. Mon Weather Rev 127: 1408-1432

Madsen H, Cañizares R (1999) Comparison of Extended and Ensemble Kalman filters for data assimilation in coastal area modelling. Int J Numer Meth Fluids 31: 961-981

Majumdar SJ, Bishop CH, Etherton BJ, Szunyogh I, Toth Z (2001) Can an ensemble transform Kalman filter predict the reduction in forecast-error variance produced by targeted observations? Q J R Meteorol Soc 127: 2803-2820

Miller RN, Carter EF, Blue ST (1999) Data assimilation into nonlinear stochastic models. Tellus, Ser A 51: 167-194

Miller RN, Ehret LL (2002) Ensemble generation for models of multimodal systems. Mon Weather Rev 130: 2313-2333

Miller RN, Ghil M, Gauthiez F (1994) Advanced data assimilation in strongly nonlinear dynamical systems. J Atmos Sci 51: 1037-1056

Mitchell HL, Houtekamer PL (2000) An Adaptive Ensemble Kalman Filter. Mon Weather Rev 128: 416-433

Mitchell HL, Houtekamer PL, Pellerin G (2002) Ensemble size, and model-error representation in an Ensemble Kalman Filter. Mon Weather Rev 130: 2791-2808

Natvik LJ (2001) A data assimilation system for a 3-dimensional biochemical model of the North Atlantic. Ph.D. thesis, Dep. of mathematics, University of Bergen/Nansen Environmental and Remote Sensing Center, NERSC, Edv. Griegs vei 3a, 5059 Bergen, Norway

Natvik LJ, Evensen G (2002a) Assimilation of ocean colour data into a biochemical model of the North Atlantic. Part 1. Data assimilation experiments. J Marine Sys In print 
Natvik LJ, Evensen G (2002b) Assimilation of ocean colour data into a biochemical model of the North Atlantic. Part 2. Statistical analysis. J Marine Sys In print

Park JH, Kaneko A (2000) Assimilation of coastal acoustic tomography data into a barotropic ocean model. Geophysical Research Letters 27: 3373-3376

Pham DT (2001) Stochastic Methods for Sequential Data assimilation in Strongly nonlinear systems. Mon Weather Rev 129: 1194-1207

Pham DT, Verron J, Roubaud MC (1998) A singular evolutive extended Kalman filter for data assimilation in oceanography. J Marine Sys 16: 323-340

Reichle RH, McLaughlin DB, Entekhabi D (2002) Hydrologic data assimilation with the Ensemble Kalman Filter. Mon Weather Rev 130: 103-114

Talagrand O, Courtier P (1987) Variational assimilation of meteorological observations with the adjoint vorticity equation. I: Theory. Q J R Meteorol Soc 113: 1311-1328

Thacker WC, Esenkov OE (2002) Assimilating XBT Data into HYCOM. J Atmos Ocean Tech 19: 709-724

Troccoli A, Balmaseda MA, Segschneider J, Vialard J, Anderson DLT (2002) Salinity adjustment in the presence of temperature data assimilation. Mon Weather Rev 130: 89102

van Leeuwen PJ (1999a) Comment on "Data assimilation using an Ensemble Kalman Filter technique". Mon Weather Rev 127: 6

van Leeuwen PJ (1999b) The time mean circulation in the Agulhas region determined with the ensemble smoother.

J Geophys Res 104: 1393-1404

van Leeuwen PJ (2001) An Ensemble Smoother with error estimates. Mon Weather Rev 129: 709-728

van Leeuwen PJ (2002) A variance minimizing filter for large-scale applications. Mon Weather Rev Submitted

van Leeuwen PJ, Evensen G (1996) Data Assimilation and inverse methods in terms of a probabilistic formulation. Mon Weather Rev 124: 2898-2913

Verlaan M, Heemink AW (2001) Nonlinearity in Data Assimilation Applications: A Practical Method for Analysis. Mon Weather Rev 129: 1578-1589

Whitaker JS, Hamill TM (2002) Ensemble Data Assimilation without perturbed observations. Mon Weather Rev 130: 1913-1924 